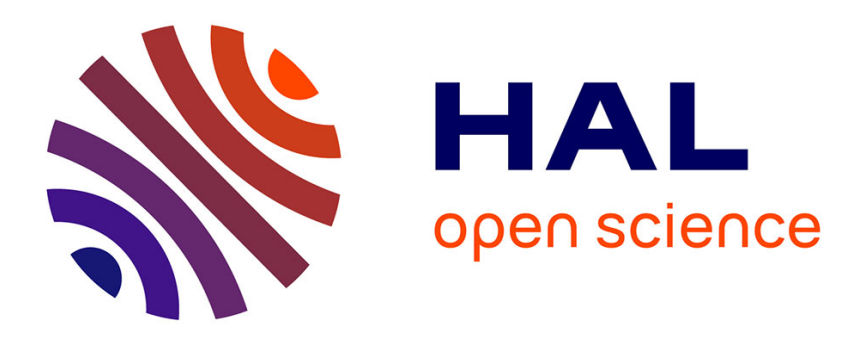

\title{
Dieu par décret. Les écritures d'un prophète africain
}

\author{
Julien Bonhomme
}

\section{To cite this version:}

Julien Bonhomme. Dieu par décret. Les écritures d'un prophète africain. Annales. Histoire, Sciences sociales, 2009, vol. 64 (nº (Culture de l'écrit en Afrique)), p.887-920. halshs-00801606

\section{HAL Id: halshs-00801606 https://shs.hal.science/halshs-00801606}

Submitted on 17 Mar 2013

HAL is a multi-disciplinary open access archive for the deposit and dissemination of scientific research documents, whether they are published or not. The documents may come from teaching and research institutions in France or abroad, or from public or private research centers.
L'archive ouverte pluridisciplinaire HAL, est destinée au dépôt et à la diffusion de documents scientifiques de niveau recherche, publiés ou non, émanant des établissements d'enseignement et de recherche français ou étrangers, des laboratoires publics ou privés. 


\section{DIEU PAR DECRET}

\section{Les écritures d'un prophète africain}

Sur les murs de Libreville, la capitale du Gabon, on peut lire d'étranges graffiti.

LE MARECHALAT DU ROI-DIEU

LE DECRET DE 1977

JESUS FERME L'ANUS AVEC SES PRIERES

PUNITION?

JUGEMENT-FOOTBALL $=$ ZERO-NEANT $=$ NEPAD 1 PLAINTE AU TRIBUNAL

\section{GOD VIVANT ANDRE ONDO MBA UN (1) DIEU VIVANT DE NOS PERES}

\section{PAPAYE (PAPE JEAN-PAUL 2) AFRIQUE AMPUTATION VOLS SACRILEGES...}

Ces graffiti sibyllins, tous écrits par un même scripteur, imposent leur présence dans le paysage urbain : certains murs en sont entièrement recouverts et on en compte des centaines en centre-ville. Ils avaient attiré mon attention dès mon premier séjour au Gabon en 2000. Mais ce n'est qu'en 2005 que j'ai croisé le chemin de leur auteur, André Ondo Mba, prophète autoproclamé. Même s'il s'est révélé être à moitié sourd et manifestement psychotique, nous avons noué une relation de confiance amicale et entamé une collaboration fructueuse. Du fait de sa surdité, notre relation passait essentiellement par l'écrit. Nous avons ainsi rempli les pages de plusieurs cahiers achetés pour l'occasion. Ce dialogue écrit m'a permis de pénétrer la logique interne de son extravagante mythologie personnelle. Outre ces cahiers écrits à deux, j'ai rassemblé d'autres types de matériaux: corpus photographique des graffiti, carnets préparatoires ayant servi à les élaborer, entretiens avec des proches, film sur Ondo $\mathrm{Mba}^{1}$. En croisant ces sources, il est alors possible de mener une étude de cas détaillée sur les graffiti et leur scripteur. Précisons d'emblée qu'il ne s'agit pas d'une étude de cas clinique: je n'envisage pas les graffiti comme une source documentaire révélatrice de la folie. Ondo Mba

\footnotetext{
${ }^{1}$ J. Bonhomme, « God's Graffiti. Prophetic agency and the pragmatics of writing in Post-Colonial Gabon », in R. BAXStrom et T. Meyers (éd.), anthropologies, Baltimore, Creative Capitalism, 2008, pp. 31-55. L'ouvrage inclut un DVD encarté avec le film God's Graffiti (26').
} 
mérite davantage que de devenir un pendant exotique du «Président Schreber », l'un des plus fameux exemples d'écrit délirant, analysé notamment par Freud ${ }^{2}$. Et de toute façon, le « fou» lui-même n'est pas hors société, ni hors culture. Il n'en reste pas moins que l'étude monographique des écrits d'Ondo Mba représente un cas-limite : elle pousse à son comble le précepte anthropologique de restitution du «point de vue indigène », puisqu'il s'agit ici du point de vue éminemment singulier d'un seul individu, fou de surcroît. Ondo Mba est un personnage si atypique qu'on ne peut le considérer comme un « informateur », représentatif d'une culture ou d'un milieu. Il ressemble en cela à Tuhami, ce Marocain marié à une djinn et vivant en marge de la société, ou encore à Menocchio, ce meunier frioulan prêchant une étrange cosmogonie et brûlé par l'Inquisition au XVI ${ }^{\mathrm{e}}$ siècle $^{3}$. Vincent Crapanzano et Carlo Ginzburg ont bien montré tout l'intérêt qu'il peut y avoir à essayer de saisir de l'intérieur la façon dont des individus atypiques pensent le monde dans lequel ils vivent. Toute singularité individuelle a en effet des limites : Tuhami et Menocchio élaborent leur vision du monde insolite à partir d'une réappropriation personnelle de ressources culturelles à leur disposition. Aussi extravagante soit-elle, la mythologie personnelle d'Ondo Mba fait ainsi appel à un imaginaire, notamment politico-religieux, qui trouve en réalité de nombreux échos dans la société gabonaise contemporaine ${ }^{4}$. On ne peut donc en saisir le sens qu'en la réintégrant dans le champ de la culture collective. Lire les graffiti de ce prophète excentrique, c'est observer le Gabon postcolonial depuis un point de vue inaccoutumé, comme à travers un miroir grossissant et déformant ${ }^{5}$.

C'est alors notamment autour de l'écriture que nous aborderons cette question des rapports entre le singulier et le collectif : celle-ci est à la fois ce qui fait la singularité d'Ondo Mba et ce qui permet de replacer ses graffiti dans une perspective plus vaste. Ce sont en effet les innombrables inscriptions publiques qu'il trace depuis vingt ans qui distinguent Ondo Mba de tous ces pauvres hères à moitié fous que l'on voit divaguer dans Libreville. Ondo Mba entretient ainsi un rapport très fort à l'écriture. Rendant possible un nouveau type de rapport à soi, mais aussi de nouvelles façons de se mettre en scène pour autrui, celle-ci constitue le moyen à travers lequel il peut élaborer les formes originales de sa subjectivité. Nous nous

\footnotetext{
2 D.P. SChreber, Mémoires d'un névropathe, Paris, Seuil, 1985 [éd. originale 1903]. S. FreUD, Le président Schreber: remarques psychanalytiques sur un cas de paranoïa (dementia paranoides) décrit sous forme autobiographique, Paris, PUF, 2001 [éd. originale 1911].

${ }^{3}$ V. Crapanzano, Tuhami. Portrait of a Moroccan, Chicago, University of Chicago Press, 1980 ; C. GInZBURG, Le Fromage et les vers : l'univers d'un meunier du XVI siècle, Paris, Flammarion, 1980.

${ }^{4}$ Sur l'imaginaire du pouvoir au Gabon, cf. J. TONDA, Le Souverain moderne. Le corps du pouvoir en Afrique centrale (Congo, Gabon), Paris, Karthala, 2005.

${ }^{5}$ Sur ce type d'approche de l'excentricité culturelle dans le domaine de la littérature, $c f$. P. Popovic, Imaginaire social et folie littéraire. Le second Empire de Paulin Gagne, Presses Universitaires de Montréal, 2008.
} 
proposons ainsi de faire l'étude monographique d'une pratique d'écriture singulière, tout en la réinscrivant au sein d'une « culture graphique » plus vaste ${ }^{6}$. Ce travail entre en ce sens dans le champ des recherches sur les «écritures ordinaires" (même si Ondo Mba revendique le pouvoir tout à fait « extraordinaire» de ses écritures) ${ }^{7}$. En marge de l'écrit officiel ou de la littérature produite par l'élite lettrée, l'Afrique connaît en effet un foisonnement créatif de pratiques populaires d'écriture, pourtant restées longtemps invisibles en raison de leur caractère souvent modeste ${ }^{8}$ : tenue d'un journal, correspondance, mémoire d'autodidacte, etc. Le graffiti fait partie de ces usages populaires de l'écrit, mais il a rarement fait l'objet d'études approfondies, car il s'agit d'une inscription souvent anonyme et éphémère. Ce n'est que lorsqu'il est pratiqué assidûment par un même scripteur qu'il acquiert une certaine visibilité. Ondo Mba n'est d'ailleurs pas totalement unique en son genre : le «graffitomane illuminé » est une figure marginale que l'on retrouve régulièrement en milieu urbain, en Afrique ou ailleurs 9 .

Une approche pragmatique centrée sur l'«acte d'écriture » est sans aucun doute la mieux appropriée à ce type particulier d'écrit qu'est le graffiti ${ }^{10}$. Il s'agit de déterminer ce que « fait» le graffiti en tant qu'écriture exposée dans l'espace urbain. Le graffiti est un écrit in situ, un texte fortement indexé à son contexte spatial et matériel ${ }^{11}$. Les inscriptions d'Ondo Mba doivent ainsi être resituées au sein de l'ensemble des signes qui les entoure et dans lequel elles prennent sens. Cette articulation entre texte et contexte doit se doubler d'une articulation entre forme et contenu. L'écriture est en effet à la fois le média de la mythologie d'Ondo Mba et l'un de ses thèmes obsessionnels. Ondo Mba est un prophète de l'écriture : il prétend accomplir la création divine à travers l'affichage public de ses graffiti. Il peut de ce point de vue être replacé dans le champ des «prophétismes scripturaires » africains, ces mouvements religieux qui accordent une place centrale au pouvoir de l'écriture ${ }^{12}$. Ses graffiti mystiques manifestent ainsi, sous une forme excessive, une idéologie de l'écriture largement partagée et dont l'origine est à chercher dans la situation coloniale, notamment dans les deux pivots du pouvoir colonial que sont la mission et l'administration. Situés au croisement du

\footnotetext{
${ }^{6}$ R. CHARTIER, « Culture écrite et littérature à l'âge moderne », Annales, 56, 4-5, 2001, pp. 783-802.

${ }^{7}$ D. FABRE (dir.), Ecritures ordinaires, Paris, P.O.L. - Centre Georges Pompidou, 1993.

8 J. FABIAN (éd.), History from below : the "Vocabulary of Elisabethville 》 by André Yav, Amsterdam Philadelphia, J. Benjamins Pub. Co., 1990 ; K. BARBER (éd.), Africa's Hidden Histories : Everyday Literacy and Making the Self, Bloomington, Indiana University Press, 2006.

${ }^{9}$ Pour un exemple brésilien de prophète graffitomane, $c f$. L. GuElman, Brasil : Tempo do Gentileza, Rio de Janeiro, Instituto Joãsinho Trinta, 2001.

${ }^{10}$ B. FRAENKEL, « Actes d'écriture : quand écrire c'est faire », Langage \& Société, 121-122, 2007, pp. 101-112.

${ }^{11}$ E. ChMiElEWSKA, « Framing [Con]text : Graffiti and Place », Space and Culture, 10, 2, 2007, pp. 145-169.

12 Sur la notion de prophétisme scriptuaire, cf. J.-L. AMSELLE, Branchements. Anthropologie de l'universalité des cultures, Paris, Flammarion, 2001, p. 65 sqq.
} 
document officiel et des Ecritures saintes, les graffiti d'Ondo Mba possèdent en outre une forte charge contestataire : ils défient les autorités en place. Toute la question est alors de savoir si Ondo Mba parvient à faire entendre son message. Nous ne pourrons donc éluder la question de la réception : comment s'articulent les prétentions extravagantes d'Ondo Mba et la réception de ses écrits publics par les lecteurs?

\section{UNE AUTOBIOGRAPHIE IMAGINAIRE}

André Ondo Mba est né en 1943 en pays fang au nord du Gabon. A l'âge de 10 ans, on l'initie au Melan avant de l'envoyer à l'école à une dizaine de kilomètres de son village ${ }^{13}$. Après avoir obtenu son certificat d'études primaires, il part à Libreville au début des années 60 , juste après l'indépendance du pays. Il se destine à une carrière de maître d'école, mais est finalement admis sur concours dans l'administration pénitentiaire. Il est affecté comme opérateur radio à la Police, avant d'être nommé brigadier à la prison centrale de Libreville à partir de 1970. Il y occupe notamment les fonctions de secrétaire dactylographe, ce qui lui donne l'occasion d'apprendre à taper à la machine à écrire et de découvrir les arcanes du Code pénal. Il se marie en 1966 et aura quatre enfants de ce premier lit. Les prodromes de sa « maladie » surviennent en 1967. Tout commence par des problèmes d'audition qu'il attribue à un acte de malveillance : alors qu'il s'était assoupi devant son émetteur radio, quelqu'un lui aurait versé du liquide de batterie dans les oreilles. Les symptômes s'accumulent au cours des années suivantes : maux de ventre, aphasie chronique. Sa femme l'emmène se faire soigner à l'hôpital et chez divers guérisseurs. Mais rien n'y fait et son état empire. Découragée, son épouse décide de divorcer en 1979. Par l'entremise de sa sœur aînée, Ondo Mba se remarie alors avec une autre femme, répudiée par son premier mari parce qu'elle ne lui avait pas donné d'enfant. Pour résoudre ce problème de stérilité, elle se fait initier au Mbiri en 1982 en entraînant Ondo Mba avec elle ${ }^{14}$. Elle tombe peu après enceinte et accouche d'un garçon, Zolo Ondo. En 1984, le couple retourne faire initier au Mbiri les quatre premiers enfants d'Ondo Mba. Selon son entourage unanime, c'est au cours de cette seconde initiation qu'Ondo Mba, qui a vraisemblablement repris une dose d'iboga, est devenu « fou ». L'iboga est réputé « ouvrir en deux » la tête de l'initié de manière à laisser sortir son esprit. Cela peut

\footnotetext{
${ }^{13}$ Le Melan est un rite de passage masculin lié au Byeri, le culte des ancêtres. L'initiation repose sur l'ingestion d'alan (Alchornea floribunda), un psychotrope végétal qui permet d'avoir des visions grâce auxquelles le néophyte peut aller au village de ses ancêtres.

${ }^{14}$ Le Mbiri (litt. « esprits ») est un rite initiatique de guérison reposant sur l'ingestion d'iboga (Tabernanthe iboga), un psychotrope végétal qui permet d'avoir des visions grâce auxquelles l'initié peut découvrir l'origine de son mal.
} 
parfois entraîner la folie lorsque le néophyte ne parvient pas à revenir de son voyage mystique et qu'il garde définitivement la "tête ouverte ». Il est ainsi probable que la prise d'un psychotrope hallucinogène ait constitué pour Ondo Mba l'événement révélateur d'une psychose latente. Depuis cet épisode, ce dernier souffre en effet d'un trouble délirant chronique $^{15}$. Il adopte en outre un comportement excentrique : il ne s'habille plus qu'en habit militaire, porte un pot de chambre en guise de casque, un faux pistolet à sa ceinture. Sa seconde épouse le quitte alors peu après. C'est d'ailleurs ce qui fait dire à ses proches que celle-ci est responsable de sa folie : des suspicions de sorcellerie familiale planent ainsi sur toute l'affaire. En 1985-86, Ondo Mba est mis en retraite anticipée en raison de sa maladie et touche depuis une petite pension d'invalidité. A la fin des années 80 , son délire se fait de plus en plus inspiré et prend une tournure nettement religieuse : il devient alors le « Maréchalat du Roi-Dieu » ou encore « God vivant André Ondo Mba ». Il adopte un nouvel accoutrement : un pantalon blanc, une chemise blanche, ainsi qu'un casque colonial (aujourd'hui remplacé par un chapeau en toile à bords rabattus). Il laisse pousser sa barbe, marque évidente de folie au Gabon car seuls les hommes en deuil doivent s'abstenir de se raser. C'est aussi à cette époque que débute sa graphomanie compulsive. Il passe des journées entières à noircir de gros cahiers, ainsi que toutes sortes de vieux papiers, paquets de cigarettes, emballages de médicaments ou reçus du Trésor public. Il va ensuite recopier ses messages partout dans Libreville. Il a d'abord commencé par écrire sur des panneaux de bois qu'il suspend aux arbres et aux poteaux. Puis il s'est mis à peindre directement sur les murs, les parapets et les pylônes. En vingt ans, il a ainsi écrit des milliers pages et autant de graffiti ou de panneaux «NTSIME » ${ }^{16}$. Ondo Mba vit aujourd'hui avec ses deux fils aînés qui restent à ses côtés pour veiller sur lui. Ce soutien lui a permis d'éviter la clochardisation qui, en ville, guette souvent les fous en l'absence de toute prise en charge psychiatrique. Ondo Mba est d'ailleurs bien inséré dans son quartier. Il fréquente voisins et commerçants qui l'acceptent tel qu'il est et lui passent ses lubies. Il accomplit en outre de petits travaux de débroussage dans son voisinage et mendie à l'occasion, afin de récolter quelques pièces qui, ajoutées à sa maigre pension, lui permettent de subvenir chichement à ses besoins.

Aussi pertinente soit-elle pour comprendre la trajectoire d'Ondo Mba, cette biographie factuelle recueillie auprès de ses proches diffère toutefois sensiblement de l'autobiographie

\footnotetext{
${ }^{15}$ S'il souffre manifestement d'une psychose, il est difficile de trancher entre une schizophrénie paranoïde tardive, une paraphrénie, une psychose hallucinatoire chronique ou même une psychose paranoïaque. La précarité et les divergences des nosologies limitent de toute façon l'intérêt d'un tel exercice diagnostic.

${ }^{16}$ « Ntsime » désigne la planche de bois en fang.
} 
imaginaire qu'il s'est lui-même construite. A travers ses écrits, il se livre en effet à une entreprise de réécriture de soi, réinterprétant toute sa biographie comme une succession de miracles. Alors que je lui demandais de me raconter sa vie, il m'a ainsi écrit :

J'ai un miracle surnaturel dans mon corps depuis 1943 l'année de ma naissance avec l'alliage des Khristos Cosmos et Créations. Un créateur et God Ondo Mba André. Après mes études primaires C.E.P.E., j’étais un élève moniteur et peu après j'étais admis au concours pour les élèves gardiens de prison. Gabonais, je suis marié divorcé et père de cinq (5) enfants. J'ai commencé la mythologie Christ, épreuve Christ Jésus et Ondo Mba à 39 ans d'âge, après avoir bouger le ciel et la terre, à 44 ans je suis allé à la limite de la corde Gaole vie coupée 2 jours seulement et Jésus Christ mort 1700 ans après 3 jours. A 50 et 52 ans d'âge j'ai fait la circoncision de l'immortel Zolo Ondo Mba NTSIME Eyola au-delà étant ici-bas un homme. La circoncision au-delà avait bien commencé en 1943 avec le nombril et en 1953 avec la première coupe des 2 prépuces du pénis Regland avec les maladies sourdes (muets) aveugles et lèpres etc... j'ai enlevé les bruits du Zénith en 1997 et 1990-1993 Dieu du ciel et de la terre à peu près : Un grand miraculeux à vie, toutes les vies ${ }^{17}$.

Une série d'événements scande ainsi l'autobiographie d'Ondo Mba. Son initiation au Mbiri marque le commencement de sa «mythologie Christ». Reprenant sous une forme excessive la phraséologie mystique typique des récits visionnaires des initiés, il raconte ainsi que l'iboga lui a permis de « bouger le ciel et la terre » et de « voir la corde des créations audelà ». Afin de ne pas détruire «l'alliage » qui fait son pouvoir surnaturel, Ondo Mba doit en outre se soumettre à toute une série d' "épreuves »: ne plus manger l'iboga, s'abstenir de toute relation sexuelle. Cette obsession angoissée de la chasteté l'amène à noter scrupuleusement ses rêves érotiques dans ses cahiers. Ce récit de vie fantasmatique relève clairement du genre de l'autobiographie prophétique que l'on retrouve couramment au sein du Bwiti fang (société initiatique dont le Mbiri est une branche) ${ }^{18}$. En adaptant le Bwiti traditionnel des Mitsogo au début du $\mathrm{Xx}^{\mathrm{e}}$ siècle, les Fang ont en effet désserré les contraintes de l'enseignement initiatique au profit de la révélation prophétique. Cette « invention d'une tradition prophétique au cœur d'une société initiatique » a ainsi donné naissance à toute une lignée de 《 petits prophètes $»^{19}$. La diffusion orale ou écrite de récits de vocation qui, tel celui d'Ondo Mba, mêlent maladies et miracles contribue alors à la légitimation du prophète. Le Bwiti fang est en outre marqué par un syncrétisme chrétien également très présent dans les

\footnotetext{
${ }^{17}$ Pour transcrire les écrits d'Ondo Mba, j'adopte la convention suivante : ses graffiti sont en capitale, les extraits de ses carnets intimes, en italique, les extraits de nos carnets ethnographiques, en romain.

${ }^{18}$ Sur Mbiri et Bwiti, $c f$. J. FeRnANDEZ, Bwiti. An ethnography of the religious imagination in Africa, Princeton, Princeton University Press, 1982, pp. 595-599.

${ }^{19}$ A. MARY, Le défi du syncrétisme. Le travail symbolique de la religion d'eboga (Gabon), Paris, EHESS, 1999, p. 111.
} 
écrits d'Ondo Mba qui, à côté de l'iboga ou du culte des ancêtres, évoquent Jésus-Christ, le Pape et la Bible. Jusqu'au début des années 1980, Ondo Mba fréquentait d'ailleurs la mission Sainte-Marie et entretenait même de très bons rapports avec l'archevêque de Libreville, Mgr Anguilé (†2001). Il se démarque toutefois du prophétisme initiatique du Bwiti fang, dans la mesure où il ne cherche nullement à inscrire sa révélation prophétique dans une communauté cultuelle. Prophète sans disciple ni mouvement, Ondo Mba se réclame cependant d'une lignée de « 210 prophètes » dont il serait le parachèvement. Tous ses prédécesseurs n'auront été que des « prophètes à mort toutes les morts ", lui seul étant un «prophète à vie toutes les vies ». Il s'identifie avec Jésus-Christ ou plus exactement avec « Jésus 3 » (le premier étant mort sur la croix et le second n'ayant été qu'un avorton). Figure prophétique de la subversion du champ religieux, Ondo Mba se place ainsi dans une relation de compétition vis-à-vis des autres religions qui ne sont que des essais avortés qu'il est censé supplanter.

\section{FIN DE JESUS ET DES MISSIONNAIRES}

Le Gabonais GVAOM des 410 pays qui remplace Jésus-Christ de Nazareth dans la Bible et Mahomet dans le Coran (nouveaux)

\section{Tout puissant}

Ondo Mba va même jusqu'à se proclamer Dieu en signant ses graffiti. A la fois signe d'identité et signe de validation, la signature atteste la présence du scripteur dans son message $^{20}$. Elle est un élément central des graffiti des lieux de pèlerinage ou des sites touristiques, mais aussi des « tags » urbains dont le message se résume à l'affichage d'un nom propre. Ondo Mba l'utilise quant à lui pour afficher publiquement son identité divine tout en conférant une autorité singulière à ses graffiti. Il accole en effet le nom de Dieu à son propre nom, manière ingénieuse de manifester son identité composite. Il signe ainsi «God vivant André Ondo Mba », formule souvent condensée par l'acronyme GVAOM, ou encore «Zame Yemebeghe Ondo Mba » (Zame-ye-Mebeghe étant l'un des noms de Dieu en fang), condensé en ZYOM. Certains graffiti se limitent d'ailleurs pour tout message à décliner à l'infini ce nom composé.

\section{LE CREATEUR GOD VIVANT AOM 1 CREATEUR GOD-DIOS A VIE TOUTES LES VIES}

EYOLA LE RENOM

DE GVAOM

\footnotetext{
${ }^{20}$ B. FrAENKEL, La signature. Genèse d'un signe, Paris, Gallimard, 1992.
} 


\section{LE NOM}

\section{ZAME YEMEBEGHE ONDO MBA, GOD VIVANT AOM}

B.P. $2110 \mathrm{LBV}$

\section{GABON}

Comme le montrent bien ces graffiti, Ondo Mba fait ainsi proliférer son nom dans la ville afin d'asseoir sa réputation divine (« eyola » signifiant justement le nom propre en fang). Le dernier exemple illustre même la volonté d'officialiser cette identité surnaturelle en affichant une adresse postale, c'est-à-dire une identité administrative (le courrier fonctionne uniquement par boîtes postales au Gabon et cela reste un petit privilège que d'en posséder une).

\section{COMMUNIQUER AVEC DIEU}

La mythologie d'Ondo Mba est structurée par une analogie entre microcosme et macrocosme, que l'on retrouve par ailleurs dans le symbolisme du Bwiti : « Toute la création est dans mon corps "), avance-t-il. Ondo Mba est Dieu créateur qui renferme en lui-même la création. Il appelle d'ailleurs la corne qu'il a sous les pieds «la corne de l'Afrique » et affirme : «Je suis San Francisco », image ingénieuse qui renvoie à la fois à la ville américaine et au saint chrétien. Ondo Mba est ainsi en contact direct avec tous les humains qui sont ses enfants, notamment par le biais des rêves : «Dieu Ondo Mba. C'est moi que vous voyez dans les rêves », m'a-t-il ainsi déclaré un jour. Au Gabon, les rêves sont un moyen par lequel les ancêtres peuvent entrer en relation avec leurs parents. Ondo Mba prend ainsi la place du premier ancêtre qui n'est autre que Dieu lui-même. S'il peut accomplir les « miracles » qui font de lui le «créateur du ciel et de la terre », c'est ainsi parce que tout ce qui lui arrive survient également dans le monde extérieur.

Lorsque les moustiques me piquent, c'est un grand miracle, ils piquent aussi tous les hommes au zénith, au-delà, dans leurs corps surnaturels et naturels.

Lorsque je coupe les herbes et buissons et les petits bois je fais toutes les créations d'un dieu, one God-Dios au Zénith et ici-bas - C'est Grave !

Ce «Zénith» désigne un espace liminal qui se situe à la fois à l'intérieur et à l'extérieur du corps d'Ondo Mba, à la limite entre l'ici-bas et l'au-delà, entre la vie et la mort. Ondo Mba l'a atteint pour la première fois au cours de son initiation au Mbiri et a ainsi pu commencer son œuvre de créateur en faisant communiquer l'au-delà et l'ici-bas. Au Zénith, Ondo Mba est en contact avec son double surnaturel qu'il surnomme : «mon immortel», 
«mon créateur », « mon homonyme spirituel », «mon ami surnaturel au-delà » ou encore «Ondo Mba des rêves ». Si un tel dédoublement de personnalité se retrouve fréquemment chez les schizophrènes, le thème du double est en réalité tout à fait banal dans les conceptions de la personne qui sous-tendent les traditions religieuses gabonaises, ce qui permet d'en nuancer l'étrangeté ${ }^{21}$. Au Gabon, on dit communément du fou qu'il «parle avec les esprits ». L'originalité d'Ondo Mba tient cependant à ce que le rapport à son double est au principe d'une identification directe à Dieu. C'est alors ce double divin qui «dicte » à Ondo Mba sa mythologie lorsqu'il « écoute au Zénith ».

Le secret de nos cœur-à-cœur lorsque nous parlons sans ouvrir la bouche et lorsque nous écoutons là-bas au-delà, s'appelle le Zénith. Au Zénith, nous entendons le créateur Dieu God qui nous parle, ainsi que nos amis, nos immortels, les vivants, les animaux, les oiseaux, les reptiles, les vers de terre, les fourmis, les insectes, les poissons qui nous parlent comme nous mêmes les hommes. Ma voix est tonnante «JUPITER ». Je parle publiquement et tout le monde entier m'entend au Zénith. Un grand miracle de Dieu (un (1) créateur God Ondo Mba).

Cette communication surnaturelle passe par deux canaux : les rêves et les voix. Ces voix sont vraisemblablement des hallucinations verbales, symptôme psychotique fréquent. La communication entre Ondo Mba et son double surnaturel constitue ainsi une projection de son propre discours intérieur (cf. sa belle expression « nos cœur-à-cœur lorsque nous parlons sans ouvrir la bouche »). Ceci explique les constantes hésitations d'Ondo Mba entre parler et écouter. Il y a en fait plusieurs actes de communication qui s'enchaînent : Dieu parle à Ondo Mba qui écrit sous sa dictée les révélations dans ses cahiers avant de les retransmettre à travers ses écrits publics. Cet enchaînement est parfois condensé en un raccourci saisissant : à l'aide d'un « haut-parleur surnaturel », Dieu Ondo Mba parle d'une «voix tonnante » qui résonne dans le monde entier. La communication surnaturelle est ainsi pensée sur le modèle de la radiotéléphonie qui permet justement d'établir une communication à distance avec un interlocuteur invisible (les graffiti mentionnent d'ailleurs les talkies-walkies). Ondo Mba se replace en effet dans la position de l'opérateur radio qu'il a été autrefois. Mais le décodage des messages est souvent entravé par les «bruits du Zénith», terme qui évoque à la fois les acouphènes qui gênent son audition et les parasites qui brouillent la communication radio. Ondo Mba transcrit ensuite les messages dans ses cahiers, dans un style souvent quasi

\footnotetext{
${ }^{21}$ L'« esprit » (nsisim en fang) désigne d'abord un double optique : l'ombre ou le reflet.
} 
télégraphique (abréviations, acronymes, phrases nominales). Et comme tout rapport de transmission au sein d'une administration, il date et signe chacun des messages officiels.

\section{DES CAHIERS AUX GRAFFITI}

L'écriture représente pour Ondo Mba un lieu crucial d'élaboration des formes singulières de sa subjectivité. On peut relever deux usages de cette «écriture de soi » en fonction de ses deux principaux supports : les graffiti et les cahiers. C'est dans le va-et-vient entre écrits privés et écrits publics, écriture pour soi et écriture pour autrui, que se joue la négociation des limites troublées du soi. C'est ce que révèle l'usage des pronoms personnels dans les écrits d'Ondo Mba. Il emploie rarement la première personne, sauf dans le récit de ses rêves qu'il consigne dans ses carnets pour essayer de leur trouver une « explication ».

Dans les rêves, j'ai fait les relations sexuelles avec une femme qui a bougé et j'ai versé. Les spermes des rêves étaient aussi publiquement.

Ondo Mba fait ainsi dans ses rêves l'expérience d'une subjectivité intime. Ses cahiers constituent en ce sens des journaux intimes (chaque entrée étant d'ailleurs datée et paraphée). Ondo Mba emploie parfois également la deuxième personne dans ses cahiers. Il s'agit là d'une projection du discours intérieur qu'il entretient avec lui-même, sans qu'il soit toutefois clair qui d'Ondo Mba ou de GVAOM s'adresse à l'autre.

Après toutes les créations, lorsque tu passes devant nous, nous te saluons.

Mbolo Ondo Mba André

\section{Vas dans ta maison}

Chez toi Ambassade de France

Ambassadeur de France pour toute la vie

Les rêves et les voix sont la matière première à partir de laquelle Ondo Mba élabore ses graffiti. Les cahiers lui servent donc aussi de carnets préparatoires, ce qui rend possible une approche génétique du texte des graffiti. Entre un rêve et le graffiti final s'intercalent en effet des dizaines de brouillons. La même phrase est réécrite avec d'infimes variations. A côté des brouillons, il note : « à revoir », « à suivre », « à reprendre » ou encore « erreur à revoir ». Lorsqu'il a enfin trouvé la bonne formulation, il note : «propre » et indique parfois le lieu en ville où il compte recopier son message. Or, en passant ainsi des récits de rêves aux écrits publics, l'usage de la première personne disparaît presque complètement au profit de la troisième personne. Il s'agit sans doute moins d'un effacement pur et simple de l'énonciateur que d'une troisième personne de majesté ou, plus vraisemblablement encore, d'une sorte de 
discours indirect libre où Ondo Mba parle au nom de Dieu. Cette situation d'énonciation paradoxale repose ainsi sur un usage singulier de la déixis pronominale : les graffiti sont signés par Ondo Mba ou plutôt GVAOM, mais mentionnent leur auteur à la troisième personne à l'intérieur du message.

\section{DIEU ONDO MBA (1) UN CREATEUR GOD A VIE AVAIT BOUGE \\ LE CIEL ET LA TERRE LES 3, 4 ET 5 JUILLET 1982 PENDANT \\ CINQ (5) ANS, A 39 ANS D’AGE (39)}

\section{NB : CELUI QUI DISPUTE LE TRONE DES MYHTOLOGIES}

DE PROPHETE A GOD VIVANT ALLAH VIVANT LE

CREATEUR VIVANT EST UN FAUT DIEU.

GVAOM

Ondo Mba s'invente ainsi une singulière voix écrite à travers ses graffiti. L'énonciateur qui prend en charge le discours n'est pas directement le sujet écrivant Ondo Mba, cet être de chair et d'os qui existe «ici-bas». C'est bien plutôt GVAOM, son « homonyme spirituel », son « immortel », c'est-à-dire une voix qui n'est justement pas faite de chair et d'os. Cette énonciation in absentia explique d'ailleurs qu'Ondo Mba reste un prophète sans disciple ni mouvement: il n'a pas fondé de nouveau culte, n'a converti personne et n'a donc pas rassemblé autour de lui quelques fidèles de la première heure susceptibles de répandre son message et de l'encadrer dans un mouvement organisé. Tout cela n'en fait pas pour autant un prophète raté. En réalité, Ondo Mba ne correspond pas à ce type spécifique d'entrepreneur religieux qu'est le prophète charismatique et c'est justement cet écart qui rend son cas si intéressant, car il permet d'interroger les catégories par leur marge. Selon l'idéal-type wébérien, le succès d'un prophète à rassembler des fidèles autour de lui tient à l'«autorité charismatique » qui est attachée à sa présence et à sa parole. La performance - notamment orale - tient ainsi une place centrale dans la manifestation de l'autorité du prophète. Or, de ce point de vue, Ondo Mba est singulièrement absent et muet : il ne prêche pas, ne guérit pas, n'accomplit aucun miracle (sa surdité l'empêcherait d'ailleurs probablement d'être un bon prédicateur). Au lieu d'être incarnée dans un locuteur de chair et d'os, la parole divine est ici intégralement dévolue à l'écrit: il s'agit bien d'une voix exclusivement écrite. Nous sommes ainsi en présence d'un message prophétique qui situation inhabituelle - fait l'économie de tout mouvement religieux mais aussi, dans une très large mesure, de la personne même du prophète. Si Ondo Mba n'est donc pas un prophète à strictement parler, j'ai néanmoins choisi de maintenir cette désignation, parce qu'il se revendique lui-même comme tel et que c'est sans doute le qualificatif qui lui convient le 
moins mal (le stigmate « fou » étant la pire option). Ondo Mba est en effet un personnage qui rentre difficilement dans les catégories constituées, celles du chercheur mais aussi, comme nous le verrons, celles de la société locale, dans la mesure où il ne fait pas l'objet d'une appréhension stabilisée par ses propres concitoyens.

On voit en définitive comment l'élaboration de soi s'opère des cahiers aux graffiti. A la fois journal intime et brouillon préparatoire, les cahiers constituent un espace de création de soi. Par contraste, les graffiti sont le lieu d'une mise en scène de soi en tant que GVAOM ${ }^{22}$. Il subsiste toutefois encore quelque chose du journal intime dans les graffiti publics. Ces derniers constituent en effet une gigantesque archive de soi à l'échelle de la ville et servent à l'occasion de support mnémotechnique à Ondo Mba. Espace intermédiaire entre les cahiers et les graffiti du centre-ville, la façade de sa propre maison est saturée d'inscriptions au point de ressembler à un extraordinaire palimpseste où s'empilent depuis des années de nombreuses couches d'écritures. Or, lorsque nous étions ensemble, il arrivait souvent qu'Ondo Mba sorte devant chez lui pour aller vérifier une date ou une formule précise. De ce point de vue, Ondo Mba ressemble fort à Rétif de la Bretonne : ce dernier avait en effet pris l'habitude de couvrir de graffiti autobiographiques les parapets de l'île Saint-Louis à Paris afin d'écrire un journal intime à ciel ouvert ${ }^{23}$. Il faisait d'ailleurs régulièrement le tour de l'île pour « commémorer» les événements évoqués par les graffiti. Pour Rétif comme pour Ondo Mba, l'espace urbain représente ainsi une «intériorité externe $»^{24}$ où peut se déployer leur subjectivité, dans une tension constitutive entre écrit intime et écrit public. Comme Rétif, Ondo Mba se livre en outre à un travail de «contrôle » de ses graffiti dont il tient le registre dans ses cahiers : il y note les inscriptions qui ont été dégradées (panneaux cassés ou décrochés, graffiti effacés ou barbouillés) en ajoutant «endommagé, à refaire », «volé le 6/6/95 contrôle », mais aussi « retrouvé et réinstallé ».

\section{LA LANGUE DES GRAFFITI}

Les graffiti d'Ondo Mba sont formulés dans un nouveau langage qu'il appelle « langage Mibeghe» (littéralement «langage de Dieu»). Cet idiolecte est en bonne partie hermétique aux lecteurs, mais Ondo Mba n'en est pas conscient et ne se soucie pas

\footnotetext{
${ }^{22}$ C'est pourquoi Ondo Mba accorde une bien plus grande valeur à ses graffiti qu'à ses cahiers, qu'il néglige au bout de quelques années, si bien que ses fils, excédés par cette accumulation de paperasse délirante, s'en débarrassent à sa place.

${ }^{23}$ N.-E. Restif DE LA BREtonne, Mes inscriptions, Paris, Plon, 1889 ; B. Fraenkel, «Écrire dans l'île. Rétif de la Bretonne, graffitomane (1734-1806) », in A. MESSERLi et R. CHARTIER (dir.), Scripta volant, verba manent. Les cultures de l'écrit en Europe entre 1500 et 1900, Schwabe, Basel, 2007, pp. 373-388.

${ }^{24}$ B. FrAenKEL, «Écrire... », art. cit., p. 387.
} 
d'expliciter ce qui devrait normalement l'être. Son langage Mibeghe repose en effet sur des substitutions lexicales qui interdisent toute interprétation littérale: par exemple, le terme « Maréchalat » signifie en réalité nombril, et « Roi-Dieu », pénis. Ce langage se réduit même parfois à une « salade de mots » typique du discours délirant : les déraillements, incohérences, stéréotypies, néologismes et autres paralogismes y abondent. Les mots peuvent s'enchaîner, non plus selon leur valeur sémantique, mais en fonction d'associations fondées sur l'allitération, l'assonance ou le rythme. S'il confine au charabia, ce déraillement du langage accouche parfois de trouvailles poétiques dont l'expressivité énigmatique fait tout l'attrait singulier des graffiti d'Ondo Mba.

\section{LES ANIMAUX DOMESTIQUES REPARLENT \\ AU ZENITH SACRILEGE}

\section{DIEUX SONT MORTS}

OPERATION CHIRURGICALEMENT FAUSSE DES PROSTATES

\section{LE COCCYX DU FAUX TRONE}

\section{LA CIRCONCISION DU SEXE DE DIEU}

\section{LES ANCETRES 7 MILLIARDS DE VICTIMES}

Il est en outre notable que le langage Mibeghe est fondamentalement un langage écrit. Pour le qualifier, Ondo Mba a d'ailleurs inventé le néologisme «ETENLANG », du verbe « eten » qui signifie "écrire» en fang. Ses écrits passent en effet par un travail sur le signifiant dans sa matérialité graphique. La disposition des signes sur la page ou sur les murs exploite toutes les ressources de l'espace graphique (saturation, listes, encadrés, décalages). Les graffiti font en outre un usage abondant de signes proprement graphiques (qui ne transcrivent pas nécessairement des marques orales) : guillemets, parenthèses, accolades, croix, points de suspension, flèches, tirets, signes d'élision pour les répétitions, soulignements, paraphes, ratures, insertions, signes arithmétiques, acronymes, tirets obliques, substitution du S par \$, répétition entre parenthèses des cardinaux en chiffres après leur mention en toutes lettres (comme c'est souvent le cas en Afrique), etc.

Le français est l'idiome majoritaire des graffiti d'Ondo Mba. Ancienne colonie française, le Gabon reste un bastion de la francophonie : le français y est la langue officielle et même la seule langue véhiculaire à l'échelle nationale. Ondo Mba participe pleinement de cette idéologie linguistique coloniale, lui qui se revendique «Le créateur Ondo Mba des 
Francophones ». Il écrit ainsi un français recherché, avec peu de fautes d'orthographe et un vocabulaire très riche, ce qui témoigne d'une remarquable maîtrise de la langue. Il a d'ailleurs une prédilection pour les termes savants (notamment scientifiques et religieux) : ontologie, archétype, didactique, paronyme, éponyme, archéologie, anthropométrie, prébende, théogonie, unicité, obédience et même «anti-anthropologie du sexe». Pour élaborer ses textes, Ondo Mba s'aidait d'un gros dictionnaire français qui l'accompagnait partout. Dans ses brouillons préparatoires, on trouve ainsi des définitions soigneusement recopiées. Un jour cependant, son fils s'est débarrassé de ce dictionnaire, après que sa sœur aînée a eu la révélation visionnaire, lors d'une initiation, que son père guérirait si ce livre encombrant disparaissait (ce qui montre bien que pour sa famille, la graphomanie est une composante essentielle de sa maladie). Ondo Mba en a été très fâché, mais n'en a pas moins continué à écrire. Les relectures obsessionnelles qu'Ondo Mba fait de ses écrits témoignent également de cette hypercorrection linguistique : il vérifie, rature, corrige, allant même jusqu'à rectifier le tracé un peu trop lâche d'une lettre. Il y a bien chez lui quelque chose du maître d'école qu'il a d'abord voulu être.

\section{PENDANT LES 30 MILLIARDS DES ANNEES FAIRE DES ECOLES APRES LA NAISSANCE DE L'HOMME NOUVEAU ZZ (Z2) POUR APPRENDRE LES NOUVEAUX LANGAGES ETATS-UNI-MIBEGHE}

Si le français prédomine, Ondo Mba emploie toutefois également des termes fang, mais aussi quelques mots anglais, souvent approximatifs : il écrit par exemple «God in strut» pour «In God we trust», ou encore «US banger » pour «US banner ». Il prétend que le langage Mibeghe inclut en outre des mots en allemand (on trouve l'occurrence « Deutsch»), espagnol («Pesetas»), italien, latin, indochinois, roumain, israélien, arabe, ainsi que des «mots de Groenlang». Le nom de Dieu est sans conteste l'objet privilégié de cette polyglossie cosmopolite: Dieu, Zame-ye-Mebeghe, God, Dios, Deum, Allah, Yahvé. Le langage Mibeghe est ainsi, selon ses propres mots, un «Espéranto », un langage universel grâce auquel il s'adresse au monde entier. Le risque est alors que ce langage universel se transforme en une cacophonie babélienne. Les voix en langage Mibeghe doivent en effet être traduites pour être comprises. Ondo Mba lui-même ne comprend pas toujours le sens des voix qu'il entend, même s'il reste persuadé qu'elles expriment des vérités supérieures. Il s'agit donc souvent pour lui aussi d'une langue étrangère. Quand je lui demandais le sens d'un terme obscur relevé dans un de ses graffiti, Ondo Mba me répondait parfois : «c'est un autre langage que je ne connais pas». Cette difficulté à déchiffrer le langage Mibeghe 
s'accompagne de l'obsession paranoïaque qu'on s'acharne à entraver sa compréhension et à brouiller la commmunication. La plupart des récits de rêves dans les cahiers d'Ondo Mba s'achèvent en effet par : « les explications ont été dérangées » ou « ces rêves ont été dérangés par les malfaiteurs ». Ondo Mba accuse même ses ennemis de «falsifier le langage courant». Cette entreprise pour trouver le sens des mots participe d'une herméneutique du dévoilement.

Les choses graves qui se cachent lesquelles nous voulons montrer le chemin au monde entier et pour toujours.

Ondo Mba vit dans un univers saturé de signes qui demandent à être interprétés. L'environnement graphique urbain constitue en effet un répertoire inépuisable de signes pour son entreprise de déchiffrement qu'il appelle fort justement une « sémiologie ».

Les sémiologies sont les inscriptions que les blancs mettent un peu partout dans les avions, les bateaux, dans les trains, dans les voitures et dans les maisons pour une enceigne etc...

Cette sémiologie est clairement liée à la signalisation urbaine et au monde des Blancs, maîtres des signes et de l'écriture. Lors de ses déambulations dans Libreville, Ondo Mba se montre particulièrement attentif à la signalétique urbaine dont il s'inspire pour la détourner dans ses graffiti. Il arpente également souvent son quartier à la recherche de «lignes surnaturelles » indispensables à ses créations. Celles-ci désignent d'anciennes rivières qui délimitent son quartier et lui donnent justement son nom. Elles renvoient ainsi au cadastre municipal, intimement associé aux Blancs, qui sont chargés de repérer puis de marquer des signes dans le paysage urbain.

Les lignes surnaturelles pour la limite de la vie et de la mort du prophète, sont créées au-delà, et si les blancs les trouvent, c'est une grande découverte scientifique, contrôlée par les autres blancs, les savants pour mettre les poteaux cadastraux et enfin pour construire leurs sémiologies (une usine, une compagnie, une société, un garage, une école etc...)

Le bornage cadastral revêt une grande importance au Gabon. Mêlant propriété coutumière et propriété moderne, le foncier est l'occasion d'innombrables litiges. Le recours à un géomètre assermenté par l'État pour le bornage des parcelles permet alors d'obtenir un titre de propriété faisant autorité. La sémiologie surnaturelle d'Ondo Mba associe ainsi la signalisation urbaine, l'autorité du document administratif et le monde des Blancs. Ce n'est d'ailleurs pas un hasard si Ondo Mba ne s'habille qu'en blanc et porte un casque colonial : il 
s'identifie lui-même à un Blanc, c'est-à-dire, selon lui, à un scientifique. Sa mythologie est en effet une entreprise autant scientifique que religieuse.

\section{Science des mythologies Christ Gouverneur Général «God» Ondo Mba André} pour les 5 continents.

Ondo Mba a d'ailleurs inventé un symbole pour désigner les « indices scientifiques » au fondement de sa sémiologie : « $m m$ » qui signifie « science avec science » (par opposition à « $m m m »$ qui signifie «science sans science »). Ces «notations pour la science de la mythologie » s'inspirent visiblement du symbolisme logico-mathématique. La technologie joue également un rôle important.

\section{SCIENCES \& TECHNIQUES \\ (PORTRAITS VIVANTS, CORPS (CREATEUR) $\mathrm{mm}$ ) JUIN 2005 \\ TOLEKIS-WALKIS 8/11/2005 \\ TELEVISION \\ \& MILLIONNAIRE}

Ondo Mba s'intéresse particulièrement aux technologies de la communication et aux appareils d'enregistrement (haut-parleur, talkie-walkie, radio, télévision, caméra). Ces appareils qui rendent possible la communication à distance (comme l'écriture) représentent pour lui des instruments mystiques permettant aux Blancs de communiquer avec Dieu enchantement de la technologie que l'on retrouve couramment au Gabon, notamment parmi les initiés.

Vous-mêmes les blancs vous cherchez Dieu Dios-Gos dans vos machines qui voient au-delà lorsque vous êtes ici-bas, c'est tout cela que vous me voyez maintenant dans les tolekis et walkis tout comme un créateur God.

Mélangeant vision et audition, Ondo Mba suggère ici que la caméra avec laquelle je le filmais permettait de révéler sa nature divine. Ma relation ethnographique avec lui s'insère en effet directement dans son prophétisme technico-scientifique. Comme il l'a déclaré à un voisin, je suis un scientifique mandaté par les Blancs pour « contrôler » ses écrits :

Le premier Blanc qui doit me contrôler ce que j'ai écrit. Ce que j'ai écrit sur les murs est important pour le Blanc. Tous les Blancs du monde. S'il y a quelque chose d'écrit sur les murs comme ça, c'est-à-dire c'est grave!

Il a d'ailleurs choisi d'intituler l'une des séries de nos cahiers ethnographiques « Les scientificités ». Ce travail de contrôle constitue pour lui un moyen de certifier ses écrits en y apposant une caution scientifique, mais aussi de les diffuser jusque dans le monde des Blancs. 
Je fais en effet partie intégrante de sa stratégie de communication. Ainsi, lorsque je lui ai demandé si j'avais moi-même une place dans sa mythologie, il m'a répondu que je devais m'efforcer d'articuler ma sémiologie avec la sienne afin d'écrire un rapport à destination de l'ensemble de la communauté scientifique :

- Dernière question : est-ce que j'ai une place, un rôle dans votre mythologie?

- Il faut supporter le poids tout comme j'ai supporté, il faut organiser votre séméologie avec les autres. L'union fait la force. Lorsque la mythologie est finie comment avoir une place avec ce qui est fini ? Faites le rapport avec les $\mathrm{mm}$ scientifiques que tous les autres Blancs pourrons bien se réjouir pendant plusieurs années.

Si un jour un article fera état de mes créations, je vous serai alors bien reconnaissant de venir me montrer cet article.

\section{LES POUVOIRS PROPHETIQUES DE L'ECRITURE}

Ondo Mba est d'abord et avant tout un prophète de l'écriture. C'est en effet elle qui permet de faire communiquer l'ici-bas avec l'au-delà. Si de nombreuses mythologies africaines affirment que la parole créatrice est à l'origine de toutes choses ${ }^{25}$, dans la mythologie personnelle d'Ondo Mba, c'est pourtant à l'écriture qu'est dévolue ce pouvoir démiurgique. L'écriture n'est pas tant un discours qui porte sur le monde qu'un acte de création à part entière. Les graffiti prétendent accomplir par eux-mêmes ce qu'ils énoncent, du seul fait de l'énoncer. Les panneaux NTSIME sur lesquels Ondo Mba écrivait au début ses messages sont ainsi de véritables « installations surnaturelles ».

Les panneaux NTSIME sont des objets naturels qui créent les choses surnaturelles (au-delà) Bible-Eyola.

Les graffiti sont donc la «création » elle-même : créer, c'est écrire publiquement le nom des choses (« eyola » désignant le nom propre en fang). La création étant un ouvrage perpétuel, l'activité d'écriture ne peut elle-même avoir de fin, sous risque d'un engloutissement du monde dans le «zéro-néant ». Ondo Mba a d'ailleurs intitulé l'un de nos cahiers ethnographiques : «ENREGISTREMENT SANS JAMAIS TESTAMENT». Comme le montre bien la mention de la Bible dans le graffiti cité précédemment, cette idéologie de l'écriture s'inspire des Écritures religieuses. On connaît l'importance accordée à l'écrit dans les religions du Livre: Dieu est présent dans les textes sacrés. Cela se traduit par

\footnotetext{
${ }^{25}$ G. CAlAme-Griaule, Ethnologie et langage : la parole chez les Dogon, Paris, Gallimard, 1965.
} 
d'innombrables usages magico-religieux de l'écriture que l'on retrouve dans l'Afrique chrétienne comme musulmane : usage divinatoire de la Bible, versets du Coran insérés dans des talismans, ingestion d'encre, etc. ${ }^{26}$ C'est alors sur le terrain de l'écriture qu'Ondo Mba se place en compétition avec les religions du Livre : ses graffiti sont censés supplanter les autres Écritures saintes.

Je suis dans les papiers avec Jésus-Christ. Nouveau Testament. Ancien Testament.

\section{LA SAINTE BIBLE EST PERIMEE AINSI QUE LE ST CORAN}

De ce point de vue, les écritures d'Ondo Mba, loin d'être un hapax, peuvent être replacées dans le champ des «prophétismes scripturaires", ces mouvements religieux qui accordent une place centrale à l'écriture en s'inspirant des religions du Livre. Le lien entre prophétisme et écriture est omniprésent dans l'Afrique coloniale et postcoloniale. Dans les années 1920 au Nigeria, au sein de l'église Aladura, le prophète yoruba Oshitelu invente une nouvelle écriture sacrée pour transcrire ses révélations visionnaires ${ }^{27}$. A la même époque, toujours au Nigeria, les fidèles d'une Église indépendante ibibio sont régulièrement pris de transes au cours desquelles ils se mettent à écrire sur le sable, des feuilles ou des écorces. En 1933, deux prophètes partent en réclusion pour parfaire cette nouvelle écriture appelée Obèri Okaimè (nom qui devient aussi celui du mouvement prophétique dans son ensemble) ${ }^{28}$. On retrouve de telles inventions d'écritures prophétiques ailleurs en Afrique, par exemple en Côte d'Ivoire, dans toute l'aire kongo, en Afrique du Sud ${ }^{29}$. Le Gabon est également concerné : Ekang Ngoua (1925-1977), l'un des prophètes du Bwiti fang, invente une écriture cryptique

\footnotetext{
${ }^{26}$ C. HAMES (dir.), Coran et talismans. Textes et pratiques magiques en milieu musulman, Paris, Karthala, 2007. L'ingestion d'encre se retrouve également hors du contexte musulman ( $c f$. J. et J.L. COMAROFF, Of revelation and revolution. Vol.1 : Christianity, colonialism, and consciousness in South Africa, Chicago, University of Chicago Press, 1991, pp. 192-193). Sur l'usage divinatoire de la Bible, $c f$. J.M. JANZEN, « Kongo Religious Renewal : Iconoclastic and Iconorthostic », Canadian Journal of African Studies, 5, 2, 1971, pp. 135-143.

${ }^{27}$ P. Probst, « The Letter and the Spirit: Literacy and Religious Authority in the History of the Aladura Movement in Western Nigeria », Africa, 59, 4, 1989, pp. 478- 495.

${ }^{28}$ R.F.G. ADAMS, «Oberi Okaime: A New African Language and Script», Africa, 17, 1, 1947, pp. 24-34 ; M.B. ABASIATTAI, «The Oberi Okaime Christian Mission: Towards a History of an Ibibio Independent Church », Africa, 59, 4, 1989, pp. 496-516.

29 J.-P. Dozon, La cause des prophètes. Politique et religion en Afrique contemporaine, Paris, Seuil, 1995, pp. 123-136; J.M. JANZEN et W. MACGAFFEY, An anthology of Kongo religion: primary texts from Lower Zä̈re, Lawrence, University of Kansas, 1974 ; J. TONDA, "Pouvoirs de guérison, magie et écriture », in J.-M. Devesa (éd.), Magie et écriture au Congo, Paris, L'Harmattan, 1994, pp. 133-147 ; E. GunNER, « Keeping a diary of visions : Lazarus Phelalasekhaya Maphumulo and the Ependale congregation of Amanazaretha ", in K. BARBer (éd.), Africa's Hidden Histories..., op. cit., pp. 155-179.
} 
pour transcrire ses révélations ${ }^{30}$. Ces écritures prophétiques, au tracé parfois plus proche du dessin que de la lettre, restent la plupart du temps indéchiffrables pour toute autre personne que le prophète lui-même (qui est d'ailleurs parfois illettré en réalité). En effet, elles ne servent pas tant à coder précisément un message pour le communiquer qu'à manifester l'autorité charismatique du prophète : ce sont en quelque sorte des « glossolalies écrites » ${ }^{31}$. Les prophétismes scripturaires font ainsi un usage iconique de l'écriture qui n'est pas sans rappeler la fameuse « leçon d'écriture » racontée par Lévi-Strauss ${ }^{32}$.

Parallèlement à ces écritures prophétiques, l'Afrique subsaharienne a vu l'émergence, au cours des $\mathrm{XIX}^{\mathrm{e}}$ et $\mathrm{XX}^{\mathrm{e}}$ siècles, de nouveaux scripts conçus pour transcrire des langues vernaculaires (syllabaires mandé, syllabaire bamoun, alphabet n'ko) ${ }^{33}$. Fortement influencés par les graphies latine et arabe, ces nouveaux scripts ont généralement été inventés en réaction à l'idéologie linguistique coloniale selon laquelle l'Afrique, continent de l'oralité, serait inapte à l'écriture. La plupart d'entre eux sont également le fruit d'une révélation prophétique : c'est Dieu, un esprit ou même un homme blanc qui révèle l'écriture à son inventeur (ce qui n'empêche pas ce dernier de passer ensuite plusieurs années à parfaire son invention). Le syllabaire bété de Bruly-Bouabré en est un bon exemple. Né en 1923 en Côte d'Ivoire, Frédéric Bruly-Bouabré a d'abord été commis aux écritures de l'administration coloniale. Au début des années 1950, suite à une révélation prophétique, il invente un syllabaire pictographique pour transcrire sa langue maternelle ${ }^{34}$. Il tente également, mais sans grand succès, de fonder un mouvement religieux, l'Ordre des Persécutés, dont il rédige les statuts sur le modèle d'un règlement colonial ${ }^{35}$. Si le syllabaire est fondé sur le bété, il s'agit en réalité pour Bruly-Bouabré d'une écriture phonétique universelle. Cet idéal d'universalité s'accompagne en outre d'une ambition encyclopédiste : dans les années 1970, Bruly-Bouabré s'attèle à une gigantesque œuvre intitulée «Connaissance du Monde » et comprenant des milliers de petits cartons qui mêlent écrit et dessin. Il y cherche obsessionnellement à

\footnotetext{
${ }^{30}$ S. ŚWIDERSKI, « Ekang Ngoua, réformateur religieux au Gabon », Anthropos, 79, 1984, pp. 627-635.

${ }^{31}$ J.M. JANZEN et W. MACGAFFEY, An anthology of Kongo religion..., op. cit., p. 27.

${ }^{32}$ C. LeVI-Strauss, Tristes tropiques, Paris, Plon, 1955, pp. 337-349.

${ }^{33}$ D. DALBY, « A Survey of the Indigenous Scripts of Liberia and Sierra Leone : Vai, Mende, Loma, Kpelle and Bassa ", African Language Studies, 8, 1967, pp. 1-51; ID., "The Indigenous Scripts of West Africa and Surinam : their inspiration and design», African Language Studies, 9, 1968, pp. 156-197 ; ID., « Further indigenous scripts of West Africa : Mandin, Wolof and Fula alphabets and Yoruba "Holy writing" ", African Language Studies, 10, 1969, pp. 161-181 ; I. DugAST et M.D.W. JEFFrEYs, L'écriture des Bamum. Sa naissance, son évolution, sa valeur phonétique, son utilisation, Mémoires de l'IFAN, 1950; J.-L. AMSELLE, Branchements..., op. cit.

34 T. MONOD, «Un nouvel alphabet ouest-africain: le bété (Côte d'Ivoire)», Bulletin de l'institut français d'Afrique noire, série B, 20, 3-4, 1958, pp. 432-553.

${ }^{35}$ D. PAulme, « Naissance d'un culte africain », Cahiers d'études africaines, 38, 149, 1998, pp. 5-16.
} 
déchiffrer des signes (qu'il appelle des « écritures ») sur les fruits, les boîtes de conserve, les scarifications traditionnelles, les drapeaux ou la signalisation maritime ${ }^{36}$. Même si Ondo Mba n'a jamais créé de nouveau script comme Bruly-Bouabré, tous deux partagent néanmoins les mêmes ambitions prophétiques et universalistes et la même fascination pour la science et l'écriture (mais aussi les signalisations). Tous deux ont en outre été des clercs de l'administration à la charnière entre les époques coloniale et postcoloniale, fonction qui a joué un rôle déterminant dans leur vocation de prophète scripturaire.

Loin d'être le reflet d'une mentalité primitive incapable de sortir des cadres d'une pensée magico-religieuse immuable, l'idéologie de l'écriture qui sous-tend les prophétismes scripturaires africains est inséparable de la situation coloniale dans laquelle elle prend sens. L'écriture n'est en effet pas une technologie cognitive neutre qui produirait partout et toujours les mêmes effets : elle s'inscrit nécessairement dans un contexte social, culturel et historique qui lui donne forme ${ }^{37}$. Technique de pouvoir autant que de savoir, l'écriture a ainsi été un instrument essentiel de la colonisation en Afrique subsaharienne ${ }^{38}$. Elle est intimement associée à ces deux pivots du pouvoir colonial que sont la mission et l'administration (notamment en Afrique centrale et australe où le script arabe a une moindre influence). Dans l'Afrique coloniale, le document écrit se confond en effet souvent avec la Bible, que l'on qualifie de «fétiche du Blanc». Dans le bassin du Congo, un même terme signifie «se convertir au christianisme » et « savoir lire ${ }^{39}$. Il est d'ailleurs notable qu'avec sa belle barbe grise et ses habits blancs, Ondo Mba ressemble à un missionnaire du temps de la colonie. Mais l'autorité de l'écrit dérive également du document officiel. L'écriture est en effet un élément essentiel des techniques de contrôle à distance des populations propres à la gouvernementalité administrative. Les sujets coloniaux ont pu faire l'expérience du pouvoir tout à fait réel de l'écriture à travers réglements et décrets, cartes d'identité et laissez-passer, amendes et procès-verbaux, cartes administratives et enquêtes statistiques, prescriptions médicales et expertises scientifiques. On comprend alors que les prophètes scripturaires aient cherché à s'approprier par mimétisme ce pouvoir hégémonique de l'écrit bureaucratique : cet enchantement de la lettre témoigne en réalité d'une compréhension lucide des ressorts de

\footnotetext{
${ }^{36}$ Ces dessins attirent l'œil de critiques d'art occidentaux, si bien que Bruly-Bouabré se retrouve exposé comme artiste « brut ». J.-H. MARTin (éd.), Les Magiciens de la terre, Paris, Centre Georges Pompidou, 1989, pp. 108109 ; Frédéric Bruly Bouabré, Paris, Réunion des musées nationaux, 1995.

${ }^{37}$ C'est le sens de la critique des travaux pionniers de Jack Goody par BRIAN V. STREET (Literacy in theory and practice, Cambridge, Cambridge University Press, 1984).

38 S. HAWKINS, Writing and colonialism in northern Ghana: the encounter between the LoDagaa and "the world on paper", 1892-1991, Toronto, University of Toronto Press, 2002.

39 E. ANDERSSON, Messianic popular movements in the Lower Congo, Uppsala, Studia Ethnographica Upsaliensia 14, 1958, p. 45.
} 
l'autorité (post)coloniale ${ }^{40}$. Les prophètes africains sont d'autant plus prompts à faire un usage rituel du document officiel qu'ils sont souvent eux-mêmes des clercs de l'administration « en rupture de comptoir ${ }^{41}$. Dans la communauté du prophète Atcho en Côte d'Ivoire, les confessions des sorciers sont transcrites par un «secrétaire», puis datées et signées comme des procès-verbaux de police. Atcho a également l'habitude de communiquer avec Dieu par « télégrammes » afin de lui transmettre l'état-civil des malades ${ }^{42}$. Les fidèles des mouvements prophétiques kongo reçoivent quant à eux un « laissez-passer » censément signé par « Simon Kimbangu, roi du Congo » ${ }^{43}$. Ce document est réputé être un «passeport pour le Ciel » ${ }^{44}$.

Ondo Mba cherche lui aussi très clairement à s'approprier l'autorité du document officiel. Ces écrits sont par exemple remplis d'une étrange comptabilité surnaturelle ${ }^{45}$.

\section{$3^{\mathrm{e}}$ MILLENAIRE \\ DOUZE (12) HEURES 12 MOIS DE L'AN $2000(57+1)=58$ \\ METAMORPHOSES LE 12-1-2000 ECRIT LE 15/2/2000 \\ AU-DELA - ICI-BAS \\ LES 3 MILLENAIRES \\ GOD OMA ZERO ROME}

Ondo Mba se livre ainsi à un calcul complexe qui traduit des années de récompense ou de punition en fonction de son obéissance aux injonctions divines. Il écrit en outre sur des manifolds, ces cahiers qui incluent du papier-carbone. Ce n'est pas un hasard, car ces cahiers autocopiants sont habituellement employés par les commerçants pour leurs factures, mais aussi par les commis aux écritures pour tout type d'acte officiel. Ondo Mba inscrit ainsi délibérement ses écrits dans le registre administratif.

\section{Les grandes puissances à Libreville au Gabon, signalent le motif de faux et usage de faux en écriture publique du Trône du Maréchalat du Roi-Dieu, God Vivant AOM mondial de Dieu Ondo Mba André, le créateur de toutes les résurrections de la mythologie et cela deux (2) fois de suite en 1997 et en 1998 pour lalala à droite.}

\footnotetext{
${ }^{40}$ Sur l'« imaginaire scriptural » comme registre constitutif des représentations du pouvoir en Afrique centrale, cf. J. TONDA, Le Souverain..., op. cit., pp. 95-100.

${ }^{41}$ C. Piault (dir.), Prophétisme et thérapeutique : Albert Atcho et la communauté de Bregbo, Paris, Hermann, 1975, p. 95.

${ }^{42}$ C. Piault (dir.), Prophétisme..., op. cit., p. 125.

${ }^{43}$ Se disant l'envoyé de Dieu sur terre pour délivrer les Noirs de la domination coloniale, Simon Kimbangu est arrêté par les autorités belges en 1921 et meurt en prison en 1951.

44 E. AndersSOn, Messianic..., op. cit., pp. 160-162. Sur l'appropriation rituelle de l'écrit bureaucratique comme moyen de résistance anti-coloniale, $c f$. aussi J.H. SMITH, « Njama’s Supper : The Consumption and Use of Literary Potency by Mau Mau Insurgents in Colonial Kenya », Comparative Studies in Society and History, 40, 3, 1998, pp. 524-548.

${ }^{45}$ Il est notable que le terme pour désigner les Blancs dans les langues du Gabon («mintangen» en fang) pourrait signifier étymologiquement «celui qui compte». La comptabilité est donc typiquement une affaire de Blanc.
} 
Les titres officiels, rangs administratifs et autres grades militaires, sont également omniprésents dans les graffiti d'Ondo Mba, lui qui se proclame d'ailleurs « Maréchalat du Roi-Dieu ».

NOUVEAU REGIME :

- HV = MARECHAL DES LOGIS

- VIH = REGISSEUR DE PRISON

- HIV = 1 HOMME DIEU DE DIEU ONDO MBA

- GIH = GREFFIER COMPTABLE

- $\mathrm{CHIH}=$ SURVEILLANT-CHEF

- GPIH = GARDIENS DE PRISON

- 5 CONTINENTS ETC... ${ }^{46}$

Ondo Mba a en outre conservé son ancien laissez-passer du temps de la sécurité pénitentaire. Il y a ajouté « Brigadier-chef, Capitaine, Général-Maréchal » et a inscrit en-tête «Le décret de 1977 ». Il s'est présenté pendant longtemps avec cette carte d'identité pour toucher sa pension au Trésor Public. Ce « décret de $1977 »$ est un événement central dans l'autobiographie imaginaire d'Ondo Mba : c'est lui qui l'a autorisé officiellement à commencer sa mythologie écrite. Il est ainsi devenu Dieu par décret.

Toutes les créations faites par le créateur God sont dedans avec tous les jours, toutes les dates et toutes les années, sauf erreur. En un mot, tout ce que Dieu fera est dans ce Décret de 1977 ou les papiers pour les mythologies Jésus et ONDO MBA.

Ce décret de 1977 est un décret international validé par l'ONU. Il porte en outre sur le langage et le pouvoir de nomination, aspect central de la mythologie d'Ondo Mba.

- Qu'est-ce que le décret de 1977 ?

- Cette loi vient des grandes puissances qui avait organisé le travail à faire des mythologies que nous faisons maintenant.

URSS

LA CHINE

ETATS-UNIS D'Amériques

la France

La Grande Bretagne

l'Espagne

Sous réserve

Du Japon

\footnotetext{
${ }^{46}$ Cette liste ésotérique laisse deviner son procédé de composition. Chaque sigle reprend les initiales du titre qui suit (sur le modèle, courant au Gabon, de l'abréviation des titres officiels) : GPIH pour Gardien de Prison, CHIH pour surveillant-CHef, GIH pour Greffier comptable, HIV pour Homme de Dieu (HV et VIH font exception). Ce jeu d'acronymes suscite en outre des connotations additionnelles : ainsi VIH / HIV qui évoque le sida.
} 
et l'INDE

observateurs

Le dossier complet de la mythologie Christ Ondo Mba du Gabon et Jésus de Nazareth d'Israël, est partie à New York au siège de l'ONU

VOIR AUSSI LES RESOLUTIONS DU

CONSEIL DE SECURITE DE L'ONU

$\mathrm{N}^{\circ} 10,11$ ET AUTRES

Les noms et les mots ne sont pas votés depuis 1945 à San Francisco en Californie du Nord aux Etats-Unis d'Amériques par les sénats et les Assemblées des 410 pays.

Les mots et noms surveillés par la Commission du drapeau Chinois pour le Trône et God.

à suivre

Les écrits d'Ondo Mba témoignent ainsi d'un imaginaire globalisé : ils évoquent plus d'une vingtaine de pays (de la Malaisie à l'Argentine en passant par les Pays-Bas), mais aussi la mort du Pape, la guerre du Liban ou encore le canal de Suez. Ondo Mba a connaissance de l'actualité internationale grâce aux titres des journaux qu'il lit lors de ses déambulations en ville. Cela montre bien que loin d'être enfermé dans un délire autiste coupé de son temps et de son milieu, il absorbe au contraire tout ce qu'il voit autour de lui pour en faire la matière première de sa mythologie. Chez Ondo Mba, l'imaginaire le plus global fait en réalité écho à l'imaginaire le plus local. La géopolitique se réplique en effet à l'échelle de son quartier. Une maison voisine est l'ambassade du Cameroun, une autre est le Groenland. L'épave d'un minibus est l'ambassade du Maroc. «Tous les pays sont là », m’a-t-il dit en me faisant visiter son quartier. Ondo Mba est également en relation avec divers personnages historiques : la Reine Élisabeth, le Président Lincoln et même le «Général» Couve de Murville qui lui a donné pour mission de ramasser du petit-bois de chauffage. L'armée française lui a également ordonné de débrousser un terrain dans son voisinage. Ondo Mba entretient en effet un rapport très particulier avec l'ancienne puissance coloniale et se déclare lui-même « ambassadeur de France ». Célébrant les cinquante ans du référendum de 1958 organisé par de Gaulle, il écrit ainsi : "Oui à de Gaulle, oui à la France, oui à l'Afrique » et parle également de «La résurrection qui s'appelle la Gaule nouvelle ».

Les papiers signés par les Français en 1947-45 etc... seront valables pour Dieu Ondo Mba le créateur God en cette année, le 5 Juin 1998 prochain pour 1999 ou l'an un (1). 18/5/98 
Les graffiti d'Ondo Mba cherchent à s'approprier l'autorité de l'écrit officiel : ce sont en effet eux-mêmes des décrets. Formulés la plupart du temps sur un mode assertif, ils accomplissent des actes de langage qui relèvent de la classe des «verdictifs » (rendre un verdict, porter un jugement sur ce qui est) ou des « exercitifs » (affirmer un pouvoir, porter un jugement sur ce qui devrait être) $)^{47}$.

\section{JESUS DEFENSE D'ADORER OU DE PRIER PARTOUT AU-DELA ICI- BAS}

\section{FAIRE L'ADMINISTRATION PENITENTIAIRE (RELATIONS SEXUELLES) DANS LA MAISON ORDRE DE NOUS-MEMES DANS LA BROUSSE LES PRESIDENTIELLES ZERO ROME (0 RHUME)}

Les créations au-delà avec le créateur Dios God Ondo Mba, sont interdites de les discuter au Gabon et dans le monde entier-

PANNEAU «NTSIME»

Le motif des saisies-arrêts des bulletins de solde des Gabonais et des autres, a été jugé dans les rêves du 5 Novembre 1996. Les raisons seront avec le créateur Ondo Mba André Allah vivant O.M.A. « God»

Ces graffiti sont ainsi des décisions surnaturelles qui supposent un ajustement du monde aux mots, plutôt que l'inverse. Leur force illocutoire est en outre bien marquée, que cela soit par le verbe ou par la signature officielle.

\section{CHANGEZ L'HEURE}

\section{$24 / 24$}

La fin du sacrement de la communion est remplacée par coupe coupe cloture coniquet

Yini iboga La décision du conseil des Ministres Gabonais du 5 et 6/7/L'an 58

Les règles de sécurité sont reconnues avec le Gouverneur général of America, Gouverneur général of URSS, Gouverneur général of Chine et Gouverneur général of France - God Ondo Mba André pour les cinq (5) continents.

C'est ainsi un «JOURNAL OFFICIEL » qu'Ondo Mba prétend afficher sur les murs, comme il l'écrit lui-même dans l'un de ses graffiti. Le fait que ses écrits soient publiquement exposés est alors un aspect déterminant de leur force illocutoire ${ }^{48}$. L'épigraphie a bien souligné le pouvoir des écritures exposées, notamment des écritures monumentales

\footnotetext{
${ }^{47}$ J.L. Austin, Quand dire, c'est faire, Paris, Seuil, 1991.

${ }^{48}$ B. FrAENKEL, « Les écritures exposées », Linx, 31, 1994, pp. 99-110.
} 
d'apparat ${ }^{49}$. Soigneusement tracés en grandes lettres capitales, les graffiti d'Ondo Mba sont faits non seulement pour êtres vus, mais aussi pour être lus. Ils se distinguent en cela des quelques « tags » qui parsèment les murs de Libreville : inventés dans les années 1960 aux Etats-Unis avant d'être largement diffusés dans les villes du monde entier, les tags sont généralement des écritures bien visibles mais peu lisibles (sauf par les tagueurs eux-mêmes) ${ }^{50}$. Ce souci de lisibilité les distingue également des glossolalies écrites des prophétismes scripturaires. Illisibles par nature, les écritures mystiques du prophète Ekang Ngoua restaient en effet inscrites dans le cadre d'une logique du secret initiatique avec laquelle les graffiti publics d'Ondo Mba rompent totalement.

La localisation est également un aspect essentiel de la pragmatique des écritures exposées. Elle contribue à la visibilité des inscriptions, mais affecte aussi directement le message. Le contexte spatial de l'inscription a en effet une fonction sémiologique (les graffiti des toilettes publiques en sont un bon exemple). Les graffiti d'Ondo Mba s'inscrivent ainsi dans un environnement graphique au sein duquel ils prennent sens. Pour l'essentiel, l'environnement graphique de Libreville se compose d'enseignes et de publicités commerciales, de fresques de prévention contre le sida, de tracts électoraux et d'affiches de propagande qui placardent le nom du Président Bongo et son portrait, mais aussi de messages religieux, tracts évangéliques et enseignes des Églises éveillées ${ }^{51}$. Sur les murs de la ville, il y ainsi une compétition graphique, souvent sauvage, entre toutes ces inscriptions. Cette concurrence spatiale engendre parfois d'étonnants télescopages entre les messages. A proximité d'un emplacement couvert des graffiti d'Ondo Mba, une publicité pour un opérateur de téléphonie mobile annonce sur un immense panneau : «BIEN AU-DELA DES MOTS ». Recouvrant un autre graffiti, l'affiche d'un opérateur concurrent montre un pasteur en pleine conversation téléphonique, ce qui laisse imaginer qu'il est en communication avec Dieu. Ailleurs, Ondo Mba inscrit un curieux message sur «LES INEXISTENCES DE EVE » juste au-dessous d'un tract évangélique qui annonce un «FESTIVAL de GUERISONS et MIRACLES. LES CEUVRES SURNATURELLES DE DIEU ». Ailleurs encore, des affiches électorales proclament: «La femme, acteur majeur du développement. Avec BONGO ONDIMBA 2005 ». Elles recouvrent à moitié une inscription d'Ondo Mba où l'on peut encore lire : « $\underline{\text { AGREE }}$ - SPERMES = ZERO. GROSSESSES = ZERO. ENFANTS $=$

\footnotetext{
${ }^{49}$ A. PetruCCI, Jeux de lettres. Formes et usages de l'inscription en Italie $11^{e}-20^{e}$ siècles, Paris, EHESS, 1993.

${ }^{50}$ A. Vulbeau, Du tag au tag, Paris, Desclée de Brouwer, 1992 ; B. FraenKel, " Graffiti : un mauvais genre », in J.-L. POUYeTO (dir.), Illettrismes et cultures, Paris, L'Harmattan, 2001, pp. 131-150.

${ }^{51}$ Sur l'environnement graphique urbain en Afrique centrale, cf. F. DE BOECK et M.-F. PLISSART, Kinshasa : récits de la ville invisible, Bruxelles, La Renaissance du livre, 2005.
} 
ZERO ». Ces échos sémantiques manifestent bien la singulière pertinence culturelle de la mythologie d'Ondo Mba.

La localisation des graffiti dans Libreville est tout aussi significative : elle obéit à une stratégie qui manifeste bien l'intelligence «situationniste» d'Ondo Mba. Ses graffiti s'étendent sur près de 25 kilomètres, depuis l'aéroport au nord jusqu'à Owendo au sud. Ondo Mba prend toutefois soin de n'écrire que dans les lieux de passage les plus visibles, avec une prédilection pour les grands carrefours. Il y a ainsi une forte concentration d'inscriptions autour de la gare routière vers laquelle converge la plupart des taxis-bus de la ville. Toutes les piles et les parapets des échangeurs de la voie express qui entoure Libreville sont également couverts de graffiti. Mais c'est surtout dans le centre-ville que se concentrent ses écrits. Le «bord de mer» est ainsi saturé d'inscriptions, depuis le Ministère des Affaires étrangères jusqu'à l'Ambassade de France. Or, la façade maritime du centre-ville constitue le cœur de l'ancienne ville coloniale et abrite aujourd'hui les lieux du pouvoir postcolonial. Depuis les débuts de la présence européenne, l'appréhension de l'espace urbain est structurée par une forte opposition entre le bord de mer, lieu de la civilisation et du pouvoir, et l'hinterland où les bidonvilles (appelés «mapanes») grignotent progressivement la forêt équatoriale. Les graffiti d'Ondo Mba dessinent par conséquent une géographie urbaine du pouvoir. Ils recouvrent les murs autour de Sainte-Marie, la plus ancienne mission catholique de Libreville, à l'endroit même où se dressait le fort d'Aumale, premier bâtiment colonial érigé en 1843. Le boulevard triomphal Omar Bongo est également couvert de graffiti. On trouve des inscriptions jusqu'aux abords du Palais présidentiel, même si Ondo Mba ne s'aventure pas à écrire sur ses murs d'enceinte. Conscient que visibilité et pouvoir vont de pair, Ondo Mba choisit donc comme cible les carrefours les plus visibles et les lieux du pouvoir politique et religieux, afin d'intensifier la force illocutoire de ses graffiti. Il cherche ainsi à s'approprier ces lieux en y apposant sa marque ${ }^{52}$.

\section{UNE ECRITURE DE LA CONTESTATION}

Les graffiti possèdent souvent une dimension contestataire et s'intègrent même parfois dans des stratégies collectives de résistance ou d'insurrection ${ }^{53}$. Disputant aux autorités le monopole sur l'écriture visible, cette «arme des faibles» dans la guerre des mots vise à

\footnotetext{
${ }^{52}$ D. LEY et R. CYBriWSKY, «Urban Graffiti as Territorial Markers », Annals of the Association of American Geographers, 64, 4, 1974, pp. 491-505.

53 J. PETEET, «The Writing on the Walls : The Graffiti of the Intifada », Cultural Anthropology, 11, 2, 1996, pp. 139-159 ; P. ARTIERES et P. RODAK, «Écriture et soulèvement. Résistances graphiques pendant l'état de guerre en Pologne (13 décembre 1981-13 décembre 1985)», Genèses, 70, 2008, pp. 120-139.
} 
conquérir un espace public dominé par un ordre graphique. Les graffiti contestataires cherchent généralement à s'approprier les pouvoirs de l'écrit officiel. C'est ce qui explique l'usage de la capitale monumentale (plutôt que de la cursive), écriture du pouvoir par excellence. Le détournement parodique des formules injonctives dans les slogans de mai 1968 est à cet égard éloquent: «LES MURS ONT LA PAROLE», «IL EST INTERDIT D'INTERDIRE », «PERMIS D’AFFICHER, LOI DU 22 MAI $1968 »$. En affichant son « JOURNAL OFFICIEL » en lettres capitales sur les lieux mêmes du pouvoir, Ondo Mba conteste lui aussi les autorités sur leur propre terrain. Ce n'est d'ailleurs pas un hasard s'il a commencé à couvrir Libreville de ses graffiti en 1988-89, au tout début du mouvement de contestation populaire qui a mené à la démocratie et au multipartisme en 1990. Au Gabon, comme ailleurs en Afrique, la contestation politique du tournant des années 90 s'est en effet traduite par une explosion d'écrits protestataires, tracts, affiches sauvages, graffiti et journaux satiriques. La critique populaire du pouvoir « bénéficie [alors] du support de l'écriture (presse, tracts et graffiti, caricature, etc.) qui lui assure une large diffusion et une pérennité que n'avait pas la dérision de l'impuissance limitée en grande partie à l'oralité ${ }^{54}$. La contestation investit ainsi les murs de la ville. De grandes fresques murales apparaissent par exemple dans tout Dakar : cette forme d'art populaire, appelée Set Setal, commence comme une entreprise de rénovation des quartiers («set setal» signifie « rendre propre» en wolof), mais devient rapidement un moyen pour la jeunesse de critiquer les élites corrompues et de se réapproprier l'espace public ${ }^{55}$. Les graffiti d'Ondo Mba participent à leur façon de ce mouvement populaire d' «insurrection par les signes ${ }^{56}$. Ondo Mba oppose radicalement religion et politique, « les choses Dieu-God» et « les choses Président de la République ». La politique n'est qu'un vulgaire simulacre de sa mythologie religieuse : une «duperie » pour «prophètes sans prophètes ». C'est pourquoi il se déclare parfois « apolitique ».

PANNEAU « NTSIME»

La politique des politiciens qui traîne avec une indignité est finie (25 ans).

24/6/95

NB : on ne prend pas God Vivant AOM avec de la politique

\footnotetext{
${ }^{54}$ C. Toulabor, « La dérision politique en liberté à Lomé », Politique africaine, 43, 1991, p. 137.

${ }^{55}$ M. Diouf, «Fresques murales et écriture de 1'histoire : le set/setal à Dakar», Politique africaine, 46, 1992, pp. 41-54.

${ }_{56}$ J'emprunte l'expression à J. BAUDRILLARD, « Kool Killer ou l'insurrection par les signes », in L'Échange symbolique et la mort, Paris, Gallimard, 1976, pp. 118-128.
} 
Il est néanmoins conscient que l'écriture est une affaire éminemment politique. Parmi ses graffiti, on retrouve en effet plusieurs fois cette belle formule qui porte ouvertement la contestation sur le terrain de l'écriture :

\section{LA DICTATURE AVEC NOS MANIERES DE VIVRE, D’ECRIRE ETC... POLITIQUE !}

$\mathrm{Au}$ moment des élections présidentielles de 1993, les premières du multipartisme, Ondo Mba écrit sur les murs de Libreville : «DEUX ROIS SE DISPUTENT LE TRÔNE AU GABON » ou encore «L'OPPOSITION GAGNE AU GABON ». Omar Bongo (au pouvoir depuis 1967) est réélu avec 51\% des voix, mais beaucoup clament que le scrutin est entâché d'irrégularités et que le Père Mba Abessole est en fait le vainqueur. Alors que la presse critique à l'égard du régime est censurée, les graffiti publics d'Ondo Mba représentent ainsi un geste de défi. Ondo Mba s'identifie d'ailleurs lui-même à l'opposition.

1 VASSAL DU SUZERAIN

ONDO MBA 3 SCIENCE AVEC SCIENCE $=m m$

L'OPPOSITION CREATEUR GOD A VIE AU GABON

Ondo Mba est particulièrement virulent à l'égard d'Omar Bongo dans lequel il voit un rival personnel.

\section{APRES BONGO'O DES PRESIDENTIELS C'EST \\ CELUI-LA GOD VIVANT AOM QUI DOIT \\ COMMANDER MAINTENANT \\ GVAOM (DEUM)}

Une curieuse coïncidence accentue encore cette rivalité. En décembre 2003, Omar Bongo décide d'ajouter le nom de son père au sien (sans doute pour anticiper des querelles de succession) et devient ainsi Bongo Ondimba. Or, la paronymie entre Ondo Mba et Ondimba est aux yeux du premier la preuve que le second n'est qu'un usurpateur (il emploie d'ailleurs lui-même le terme " paronyme »). Il déclare ainsi à son égard : "il n'est même pas dans mes papiers ». Il se plaint du «faux trône avec l'emprunt du président Bongo » et accuse ce dernier de vouloir tuer son « immortel ».

ONDO MBA est un alliage 3 Khristos

cosmos

créators

à vie

tandis que ONDIMBA est un faux Dieu sans l'alliage 3

sans les créations 
sans supporter le célibataire 8 ans

et qui veut toujours disputer le trônes et les créations de l'autre sans faire pour lui-même (le camp des autres gardiens de prison)

\section{NB : LE FAUX DIEU S’APPELLE EN ALLEMAND : ONDIMBA}

Omar Bongo a raconté que c'est son père qui lui était apparu en rêve pour lui demander d'ajouter son patronyme (stratégie courante pour justifier un changement au nom de la volonté des ancêtres). Ondo Mba s'est emparé de l'histoire pour affirmer que c'est en fait de ses propres rêves que ce nouveau nom est sorti : «ONDIMBA est venu des rêves de nos songes le 27 Décembre 2003 ». Le président Bongo Ondimba ne serait donc que l'une de ses créations. Il déclare même qu'il est en réalité une partie résiduelle de son propre corps, le prépuce ou l'hymen selon les versions.

Président Bongo Ondimba est un membre de mon corps incirconcis, circoncis maintenant avec notre génération des 63 ans d'âge - 1 du créateur

La paronymie induit ainsi une troublante proximité qui va même jusqu'à l'identification paradoxale à travers l'invention du nom composé «BONGO ONDO MBA » ou encore le détournement acronymique d'OMAR qui devient « Ondo Mba André Roi-dieu coupé ». Tout cela fait d'Ondo Mba le double halluciné du Président Bongo. Dans ses graffiti, Ondo Mba tourne alors en ridicule le président usurpateur : il déclare qu'il n'est plus qu' « exPrésident de la République Gabonaise », le qualifie de «Président à mort», inversion caustique du «Président à vie » qu'il est depuis 1967. Il raille également Bongoville, le village natal du président pompeusement rebaptisé. Il lui a même rédigé une épitaphe publique sur un mur décrépi.

CI-GIT ONDIMBA $1 \stackrel{\text { ER }}{\text {-2-2005 }}$

BONGOVILLE

EPAVE DU BATEAU

EKUM Z. BONGO ONDIMBA = BONGO ZERO BZ. UNE MALCHANCE.

ALLIANCE ABIMEE-FOUTUE

S'APPELLE BONGO GOS-GOS

$m m m$ SCIENTIFIQUES DE JESUS-CHRIST

POURRITURES DE ONDIMBA 
Bien qu'il vandalise les murs de Libreville depuis vingt ans et qu'il se livre à des outrages publics contre les autorités, Ondo Mba n'a jamais été inquiété (alors que la presse fait parfois encore les frais de la censure). C'est son statut de «fou » qui le protège, comme l'explique son fils aîné : «Les autorités ne l'ont jamais embêté. Ils savent qu'il n'est pas normal. Si tu viens pour parler à un fou, c'est que quelque part toi-même tu es fou. Ils sont obligés de laisser. » Ondo Mba joue en quelque sorte le rôle du fou du roi qui peut proférer impunément les vérités les plus violentes. L’un de ses graffiti, «CROWN CLOWN», souligne d'ailleurs avec mordant qu'une seule lettre sépare le monarque de son simulacre bouffon. Ne nous leurrons pas cependant : Ondo Mba ne reste en liberté que parce qu'il n'est qu'un inoffensif excentrique dont les protestations délirantes ne peuvent sérieusement menacer les intérêts du régime. S'il n'a jamais été inquiété, certains de ses graffiti font néanmoins l'objet de dégradations volontaires : des lettres ont été effacées tandis que d'autres ont été rajoutées, de manière à rendre illisible le message. Si l'auteur de ces caviardages et ses motivations restent inconnus, leur caractère systématique témoigne en tout cas de la saillance des graffiti d'Ondo Mba dans l'espace urbain. Une partie des graffiti est en outre périodiquement effacée par les services municipaux, généralement peu avant le 17 août, jour de fête nationale qui commémore l'indépendance. A cette occasion, les autorités entreprennent en effet des travaux de rénovation afin de redorer l'image du régime. Il y d'ailleurs quelque chose d'ironique à observer un peintre municipal avec son rouleau au bout d'une perche peiner pour effacer le haut d'un mur sur lequel Ondo Mba a écrit « JOURNAL OFFICIEL ». Même s'il ne tarde pas à recouvrir ces murs vierges de nouveaux écrits, Ondo Mba s'insurge contre le 17 août et la «secte des indépendances de 1960 », preuve de l'acharnement du pouvoir contre lui.

Question: Pourquoi les fêtes du 17 Août de chaque années sont-elles contre mes écrits qui sont publiques pour toutes mes justifications?

La résolution 138 du conseil de sécurité de l'O.N.U. et du Gabon de 168.000 $\mathrm{km}^{2}$, est annulée de plein droit lorsque les panneaux de la résolution pour les mythologies-Christ, sont abîmés par la secte des indépendances de 1960.

Il suspecte également les autorités de modifier subrepticement ses graffiti afin de diffuser une « fausse mythologie ».

Ils m'ont effacé beaucoup et pour mettre les fausses dates ainsi pour faire les fausses rédactions 
C'est pourquoi il entend porter plainte contre le régime. Ondo Mba écrit en définitive des graffiti pour protester contre l'effacement de ses graffiti.

\section{PLAINTE FAUSSES MYTHOLOGIES}

Ils avaient cassé mes constructions ; la hutte de la mythologie et la maison du PK 12,900 c'était pour cela que j'avais rendu publique mes écrits pour une plainte mondiale.

Ma plainte est publique pour et contre les fausses et bonnes mythologies. Ils payeront l'amende pour 300.000 Dolars Américains paraît-il au zénith.

\section{LA RECEPTION DES ECRITS}

Ondo Mba prétend s'adresser au monde entier à travers ses graffiti.

- A qui s'adressent vos inscriptions?

- Aux 5 continents en vertu du décret de 1977 pour la mythologie dans le monde entier.

\section{GOD ONDO MBA ANDRE 5 CONTINENTS}

Il y a cependant un décalage criant entre les prétentions démesurées d'Ondo Mba et la réalité à laquelle elles se heurtent nécessairement. Ses graffiti supposent en effet une conception magique de la performativité langagière. Pourtant, le pouvoir des mots n'est jamais que virtuel : toute prétention illocutoire reste vaine tant qu'elle n'est pas suivie d'effets perlocutoires, c'est-à-dire a minima tant que l'intention communicative n'est pas reconnue par un tiers. Un message écrit représente ainsi un point d'intersection virtuel entre un scripteur et un lecteur ${ }^{57}$; mais dans le cas des graffiti d'Ondo Mba, l'existence même d'une telle intersection est incertaine, tant l'écart paraît grand. Afin de lever tout soupçon de complaisance envers les élucubrations d'un vieux fou, nous ne pouvons donc éluder la question de leur réception. Le fait qu'Ondo Mba n'ait pas de disciple ne signifie pas pour autant qu'il n'ait pas de lecteurs. Mais les écrits publics d'Ondo Mba sont-ils vraiment lus ? Et comment sont-ils lus? Les graffiti font habituellement l'objet d'une lecture flottante caractéristique des modes de perception de l'environnement graphique urbain: on les remarque en passant, plus qu'on ne les lit. Il suffit de se poster dans le centre-ville de

\footnotetext{
${ }^{57}$ R. HARRIS, La sémiologie de l'écriture, Paris, éd. CNRS, 1993.
} 
Libreville pour vérifier que les écrits d'Ondo Mba ne font pas exception ${ }^{58}$. Tout le monde connaît pourtant Ondo Mba de vue ou de réputation : il est une figure publique que l'on identifie habituellement à sa graffitomanie, à ses habits blancs ou à l'une de ses signatures (« le Maréchalat du Roi-Dieu»). Cela n’implique toutefois pas nécessairement que ses graffiti soient lus en détail, ni surtout qu'on les comprenne. C'est là tout leur paradoxe : l'efficacité des moyens pragmatiques mis en œuvre pour assurer leur visibilité contraste fortement avec leur opacité sémantique. Les graffiti mêlent jusqu'au vertige tous les registres de sens : cette créativité débridée excite l'imagination des lecteurs, au risque cependant de sombrer dans le néant de l'incommunicabilité du fait même de cette surcharge sémantique. Les graffiti suscitent en effet généralement la perplexité des lecteurs les plus attentifs. "S'il est un opposant politique, s'il n'est pas un opposant politique, je ne peux pas le déterminer. Parfois, il parle de religion, parfois il parle de politique. Alors j'ai du mal à déterminer ce qu'il pense », comme me le confiait un voisin d'Ondo Mba, enseignant retraité. Pour beaucoup, ces graffiti incompréhensibles sont l'œuvre d'un «fou ». Au Gabon, la folie est souvent associée à la sorcellerie (mais le prophète et l'initié sont toujours aussi un peu sorciers) et au pouvoir dont elle est le revers obscur. La rumeur raconte par exemple que l'épouse du président gabonais, décédée à 45 ans des suites d'une maladie, a en réalité succombé à la folie provoquée par une trop intime fréquentation du pouvoir. La folie est en effet communément perçue comme la conséquence d'une transgression d'interdit, qui est elle-même estimée être un mal nécessaire pour accéder au pouvoir. Cette association inquiétante entre folie, pouvoir et sorcellerie pousse nombre de personnes à se tenir prudemment à distance d'Ondo Mba et de ses graffiti. Un jeune homme du voisinage, perplexe devant les façades graffitées de sa maison, me confiait ainsi : «Je ne vais pas lui poser de questions. Ça fait peur. Les Africains ont peur des fous. On ne sait pas pourquoi il est devenu comme ça. Peut-être il a pratiqué, il a touché des choses, des médicaments qu'il ne fallait pas. »

Cette aura sorcière qui entoure Ondo Mba confère également à ses graffiti une valeur occulte. D'aucuns voient en effet en lui le détenteur d'un mystérieux savoir lettré. « C'est un savant », ai-je souvent entendu dire. Certains prêtent même une valeur prémonitoire à ses révélations prophétiques. Son fils, qui le décrit pourtant habituellement comme un dérangé, affirme ainsi : "Quand il commençait à écrire sur les murs, il avait écrit des choses qui devaient arriver. C'était même passé à la télé. Il avait écrit : "Deux rois se disputent le trône au Gabon". Une année après, on voit Bongo et Mba Abessole se battre. C'était lors des

\footnotetext{
${ }^{58}$ En 2007 , le taux d'alphabétisation des plus de 15 ans était de $86 \%$ au Gabon. Tout quidam ou presque est donc capable de lire les graffiti d'Ondo Mba.
} 
premières élections en 1993. »Ces mystérieuses révélations suscitent ainsi l'attention de «grands types », expression consacrée qui désigne les personnes occupant une position sociale importante. «C'est un intelligent. C'est un monsieur. Les choses qu'il écrit, il y a beaucoup qui ont cherché à savoir. Des grandes personnes qui veulent savoir ce qu'il fait. Tout le temps, on demande », comme me l'a confié sa première femme. Et son fils aîné de confirmer : «Vers les années 1998-99, Papa recommençait à écrire fort. C'est une période où les journalistes ont commencé à poser les questions, à faire des petits reportages sur ses écritures. » Journalistes, intellectuels, étudiants et peut-être même pasteurs et politiciens, ont ainsi cherché à rencontrer Ondo Mba pour s'enquérir du sens de ses écrits. Des articles de presse et même un petit reportage du journal télévisé de la chaîne nationale ont évoqué le sujet. Ondo Mba a donc réussi à acquérir une certaine notoriété à travers ses graffiti.

L'un de mes amis, Auguste Moussirou-Mouyama, écrivain, éditeur et sociolinguiste à l'Université Omar-Bongo, a même écrit au début des années 90 une pièce de théâtre intitulée « Le Maréchalat du Roi-Dieu » qui s’inspire directement des graffiti d'Ondo Mba. Dédiée « à André Ondo Mba, poète perdu à travers les poteaux d'une ville qui se voulait libre », cette œuvre est une satire ubuesque du pouvoir postcolonial, un peu dans la veine d'un Sony Labou Tansi $^{59}$. Par un étrange concours de circonstances, la pièce, pourtant inédite, a été mise au programme officiel des festivités du 17 août 2006 et devait être jouée devant un parterre de personnalités. La représentation a toutefois été annulée au dernier moment. Les deux années suivantes, la pièce devait de nouveau être jouée lors de la Fête de la Culture ; mais là encore, la représentation n'a pas eu lieu, la pièce ayant été jugée trop subversive. Il y a assurément quelque ironie à retrouver les écrits contestataires d'Ondo Mba au programme officiel de la fête nationale, le ballet des programmations et déprogrammations de dernière minute ajoutant encore au caractère grotesque de l'affaire. Mais la postérité des graffiti d'Ondo Mba n'est pas seulement littéraire. En mars 1991, l’hebdomadaire international Jeune Afrique consacre un dossier spécial au tout récent processus de démocratisation au Gabon. Le dossier s'ouvre ainsi $^{60}$ :

En Afrique, les langues se délient et les murs commencent à parler. Un peu partout des graffiti irrévérencieux, autrefois inimaginables, s'étalent sur les

\footnotetext{
${ }^{59}$ L'épithète « ubuesque » est appropriée : au Gabon, Omar Bongo Ondimba est parfois appelé « Père OBO », sobriquet moqueur qui joue sur ses initiales. Ce détournement des acronymes est typique de la subversion du pouvoir par la dérision en Afrique. Il est notable qu'Ondo Mba s'adonne lui aussi avec passion à ce genre de jeu sur les mots.

${ }^{60}$ P. GIRARD, «La moins ingrate de toutes les solitudes», Jeune Afrique, n $1575,6-12$ mars 1991, dossier spécial « Gabon. Une démocratisation réussie ?», p. 40.
} 
façades des immeubles, les parapets des ponts ou les rambardes des quais et des jetées. Libreville n'échappe pas à la règle. A faible mais prudente distance du palais Rénovation, face à l'océan, une main vengeresse dénonce le "maréchalat du roi-dieu", entendez le chef de l'Etat, que l'hebdomadaire La Griffe qualifie volontiers de "Zeus trônant sur l'Olympe".

Quelques pages plus loin, une interview avec le président Bongo revient sur ces graffiti $^{61}$ :

Jeune Afrique: L'hebdomadaire La Griffe vous qualifie de "Zeus trônant sur l'Olympe", un graffiti sur les murs de Libreville vous appelle "le roi-dieu", un terme dont les Français usent pour qualifier leur président. Comment réagissezvous?

Omar Bongo: Africain et musulman, je suis bon croyant et sais que je ne suis pas Dieu. Comme démocrate, je sais que c'est le peuple qui est roi. Au Gabon, je suis le premier parmi ceux qui ont en charge les affaires du peuple. C'est une fonction très importante : très souvent, en dernier ressort, il me revient de dire et de faire ce qui est nécessaire par-delà les visions, les schémas, les revendications des différents groupes, catégories ou clans. C'est sans doute cela qui isole les chefs d'Etat de leurs concitoyens. Pour ma part, je ne m'enferme pas dans ma fonction. Cela n'est ni dans ma nature, ni dans mon éducation, ni dans mon style de gouvernement.

Ondo Mba a eu connaissance de cette interview et y a répondu par un nouveau graffiti qui indique la référence du numéro de Jeune Afrique et rétorque qu'il est le seul à commander au Gabon. Ce dialogue par personne interposée entre Ondo Mba et Omar Bongo est intéressant à plus d'un titre. Il confirme la remarquable saillance des graffiti, mais aussi leur pertinence politique. Aux yeux du journaliste, cette inscription (dont il ignore l'auteur) résume l'esprit de la contestation du début des années 90, événement décisif de l'histoire récente du Gabon et, plus largement, de toute une partie de l'Afrique. Le journaliste met en outre sur le même plan le graffiti d'Ondo Mba et la presse satirique gabonaise. Tous deux partagent en effet le même sens de l'outrance et de l'ironie dans la formule - cet art de la dérision corrosive qui constitue plus généralement le registre favori de la critique populaire du pouvoir en Afrique ${ }^{62}$. Les écrits d'Ondo Mba font ainsi écho à la presse satirique qui s'inspire elle-même directement de la rumeur populaire de la «radio-trottoir » et de ses excès ${ }^{63}$. Ces échos stylistiques et sémantiques font des graffiti d'Ondo Mba une caisse de résonance

\footnotetext{
${ }^{61}$ «En démocratie, le multipartisme est une technique », propos recueillis par P. GIRARD, Jeune Afrique, op. cit., p. 50 .

${ }^{62}$ C. TOUlABOR, « Jeu de mots, jeu de vilains. Lexique de la dérision politique au Togo », Politique africaine, 3 , 1981, pp. 55-71 ; ID., « La dérision politique en liberté à Lomé », art. cit.

${ }^{63}$ Sur le style de la presse populaire africaine, cf. M.-S. FrERE, Presse et démocratie en Afrique francophone, Paris, Karthala, 2000, pp. 255-303.
} 
collective. Le titre baroque de «Maréchalat du Roi-Dieu » est en effet une belle trouvaille. Il évoque le Maréchal Mobutu Roi du Zaïre, mais aussi Bokassa, autoproclamé Maréchal et Empereur de Centrafrique, ou encore Idi Amin Dada, Maréchal, Roi d’Écosse et Conquérant de l'Empire britannique. Il fait en outre allusion à la tonalité prophétique du pouvoir politique en Afrique ${ }^{64}$. Mobutu, Eyadéma ou Houphouët-Boigny se dépeignaient souvent eux-mêmes en messie. Macías Nguema se proclamait quant à lui «miracle unique de la Guinée équatoriale » et déclarait : « No hay más Dios que Macías », jouant sur la paronymie entre son nom et Mesías (« messie » en espagnol). Le titre de « Maréchalat du Roi-Dieu » parvient ainsi à condenser en une formule saisissante les trois registres constitutifs de l'imaginaire postcolonial du pouvoir en Afrique subsaharienne : le pouvoir militaire, un pouvoir politique autocratique et le pouvoir religieux. Le malentendu du journaliste de Jeune Afrique est d'ailleurs révélateur: il suppose que le graffiti du «Maréchalat du Roi-Dieu » est une dénonciation satirique du despotisme du chef de l'Etat gabonais, alors qu'il s'agit en réalité de l'un des noms qu'Ondo Mba se donne lui-même. L'intimité troublante, que révèlent les graffiti, entre Ondo Mba et Bongo Ondimba, entre le pouvoir et son simulacre, n'est donc pas qu'un pur fantasme du premier, puisqu'elle se retrouve jusque dans l'interprétation des lecteurs. Mais comme Achille Mbembe l'a bien montré, une même esthétique du grotesque caractérise aussi bien l'exercice du pouvoir postcolonial que sa caricature subversive : «Dans une large mesure, les éclats de l'hilarité ou de la raillerie populaires ne font que prendre le monde officiel au sérieux, c'est-à-dire tel qu'il est en réalité et tel qu'il se représente ${ }^{65}$. A l'outrance baroque des graffiti du Maréchalat du Roi-Dieu fait ainsi écho l'ironie grotesque de la programmation officielle de la pièce de théâtre éponyme. Et c'est bien là ce qui fait tout le succès des graffiti d'Ondo Mba, aussi extravagants soient-ils : ils donnent à lire sur un mode outrancier un imaginaire du pouvoir et de l'écriture qui est bien plus partagé qu'on ne le pense.

\footnotetext{
${ }^{64}$ J.-F. BAYART, L'illusion identitaire, Paris, Fayard, 1996, pp. 117-120.

${ }^{65}$ A. MBemBe, De la postcolonie: essai sur l'imagination politique dans l'Afrique contemporaine, Paris, Karthala, 2000, p. 147.
} 


\section{Dieu par décret. Les écritures d'un prophète africain}

Résumé : Cet article s'intéresse à d'étranges graffiti qui couvrent les murs de Libreville (Gabon). Leur scripteur, André Ondo Mba, est un personnage excentrique, prophète autoproclamé qui prétend accomplir la création divine à travers ses écritures publiques. Ses graffiti manifestent, sous une forme excessive, une idéologie de l'écriture dont l'origine est à chercher dans la situation coloniale, notamment dans les deux pivots du pouvoir colonial que sont la mission et l'administration. Situés au croisement du document officiel et des Ecritures saintes, les graffiti d'Ondo Mba possèdent en outre une forte charge contestataire : ils défient les autorités en place. Toute la question est alors de savoir si Ondo Mba parvient à faire entendre son message. L'article s'intéresse ainsi à la réception des graffiti. Aussi extravagante soit-elle, la mythologie personnelle d'Ondo Mba fait appel à un imaginaire, notamment politico-religieux, qui trouve en réalité de nombreux échos dans la société gabonaise contemporaine.

\section{God by decree. The writings of an African prophet}

Abstract: This article deals with odd graffiti covering the public walls of Libreville (Gabon). Their writer, André Ondo Mba, is an eccentric character, a self-proclaimed prophet who claims to perform the divine creation through his public writings. His graffiti reveal, in an excessive manner, an ideology of writing the origin of which lies in the colonial situation and its two main pillars, mission and administration: they are indeed Holy Scriptures and official documents at the same time. Ondo Mbas' graffiti are also protest writings: they challenge the authorities. But does Ondo Mba succeed in communicating his message to the public? In order to answer this question, the article examines the readers' reception of the graffiti. Ondo Mba's personal mythology, though extravagant, resorts to a political and religious imagination which finds in fact many echoes in postcolonial Gabon. 


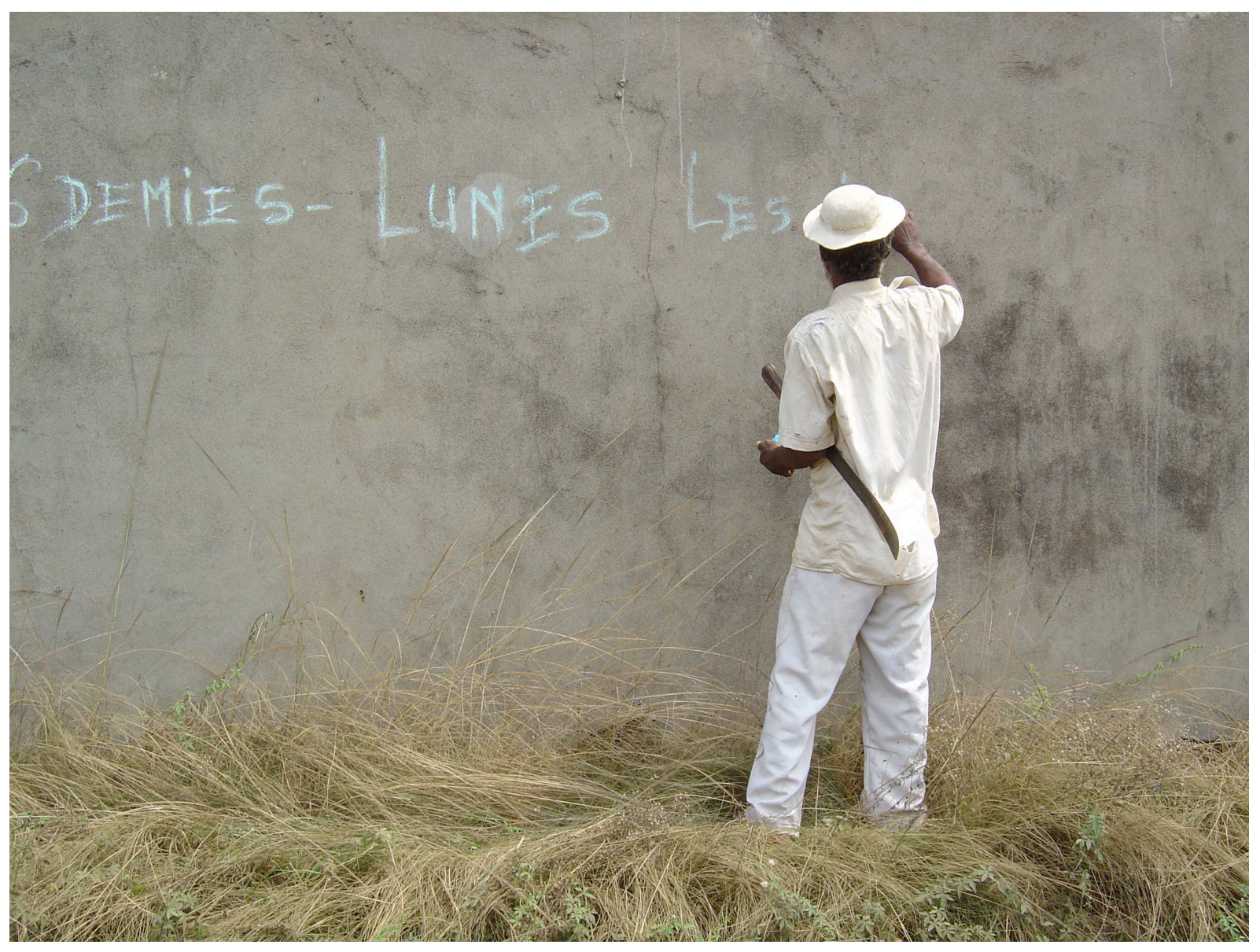




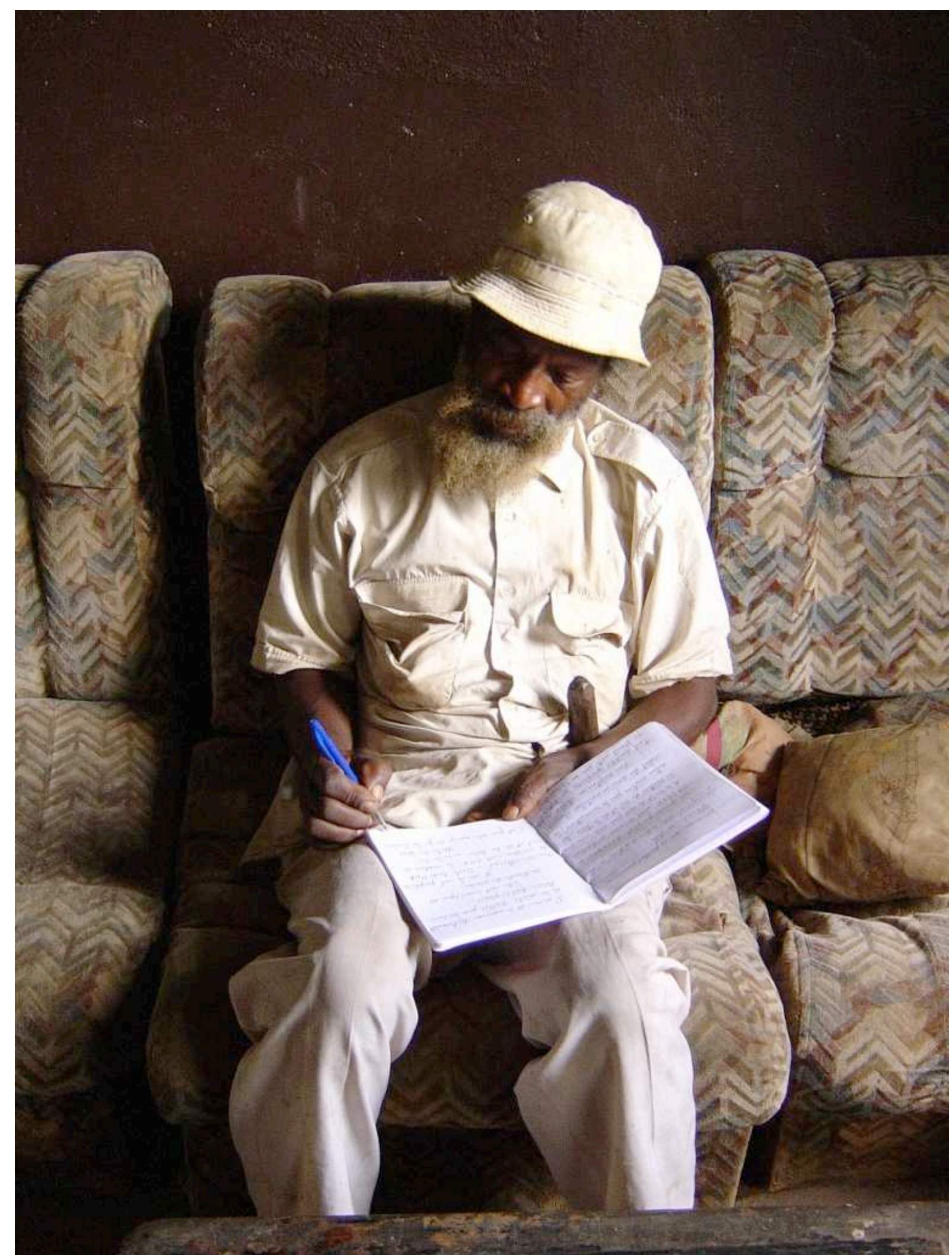




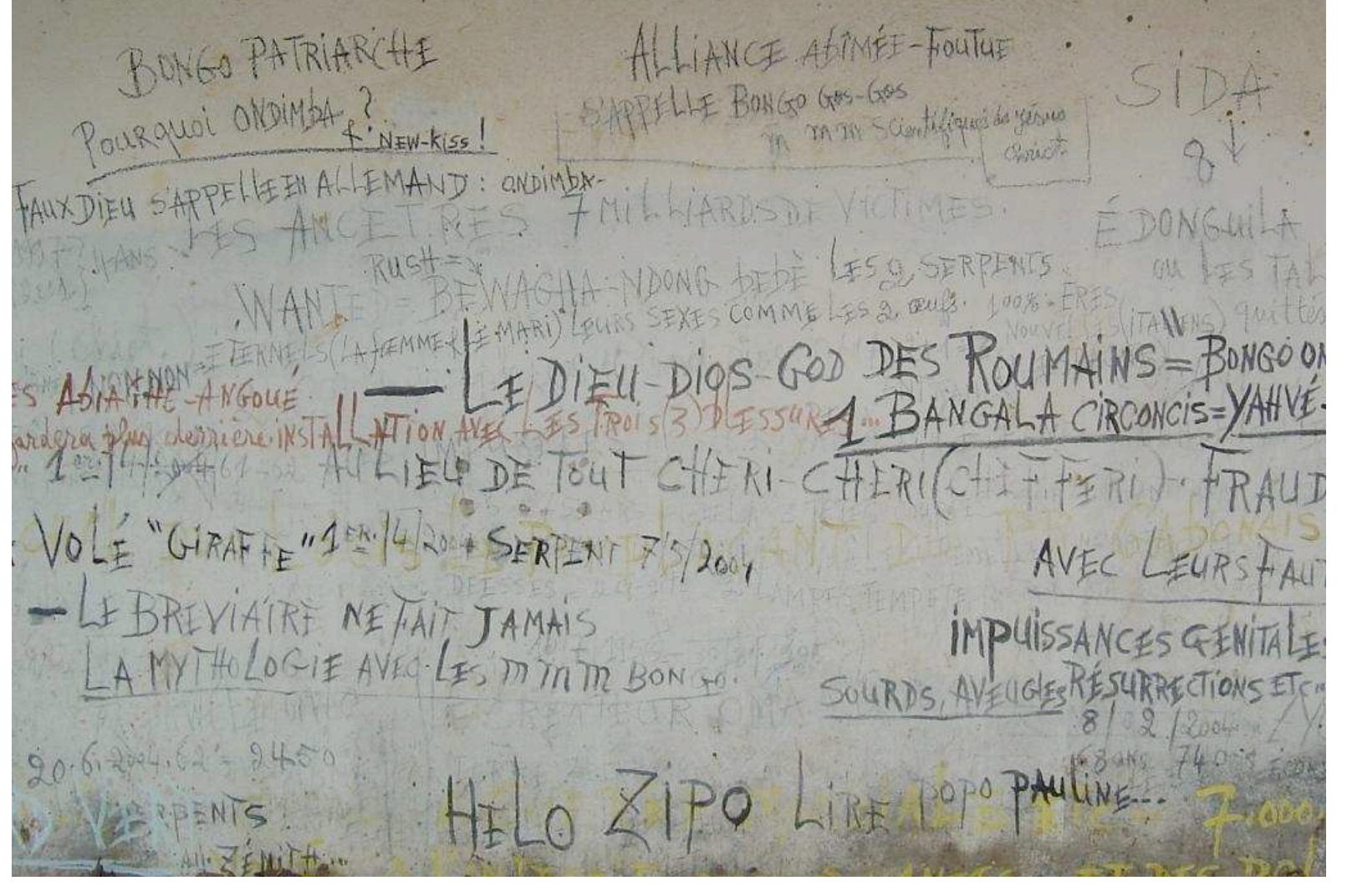

39 


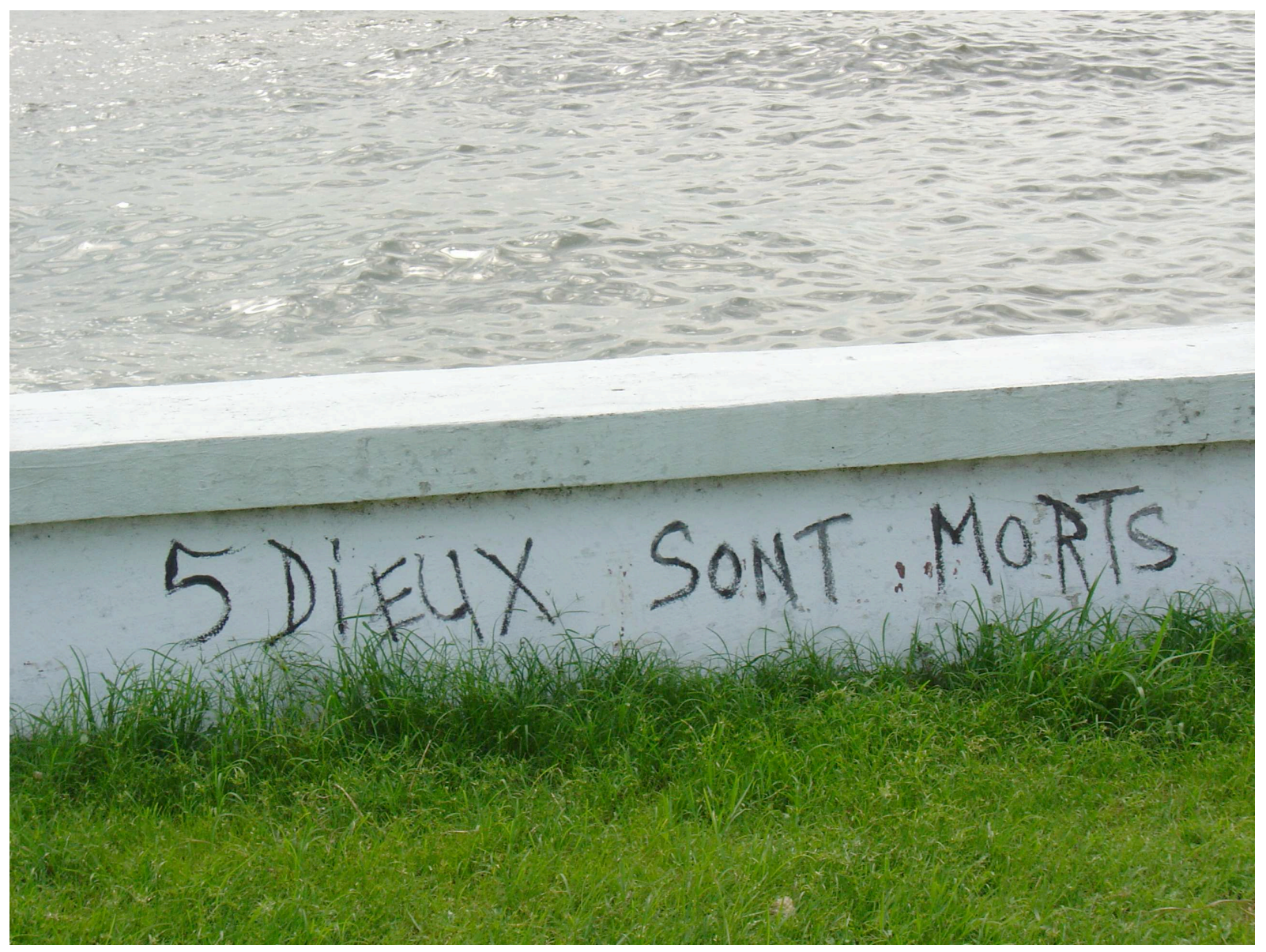




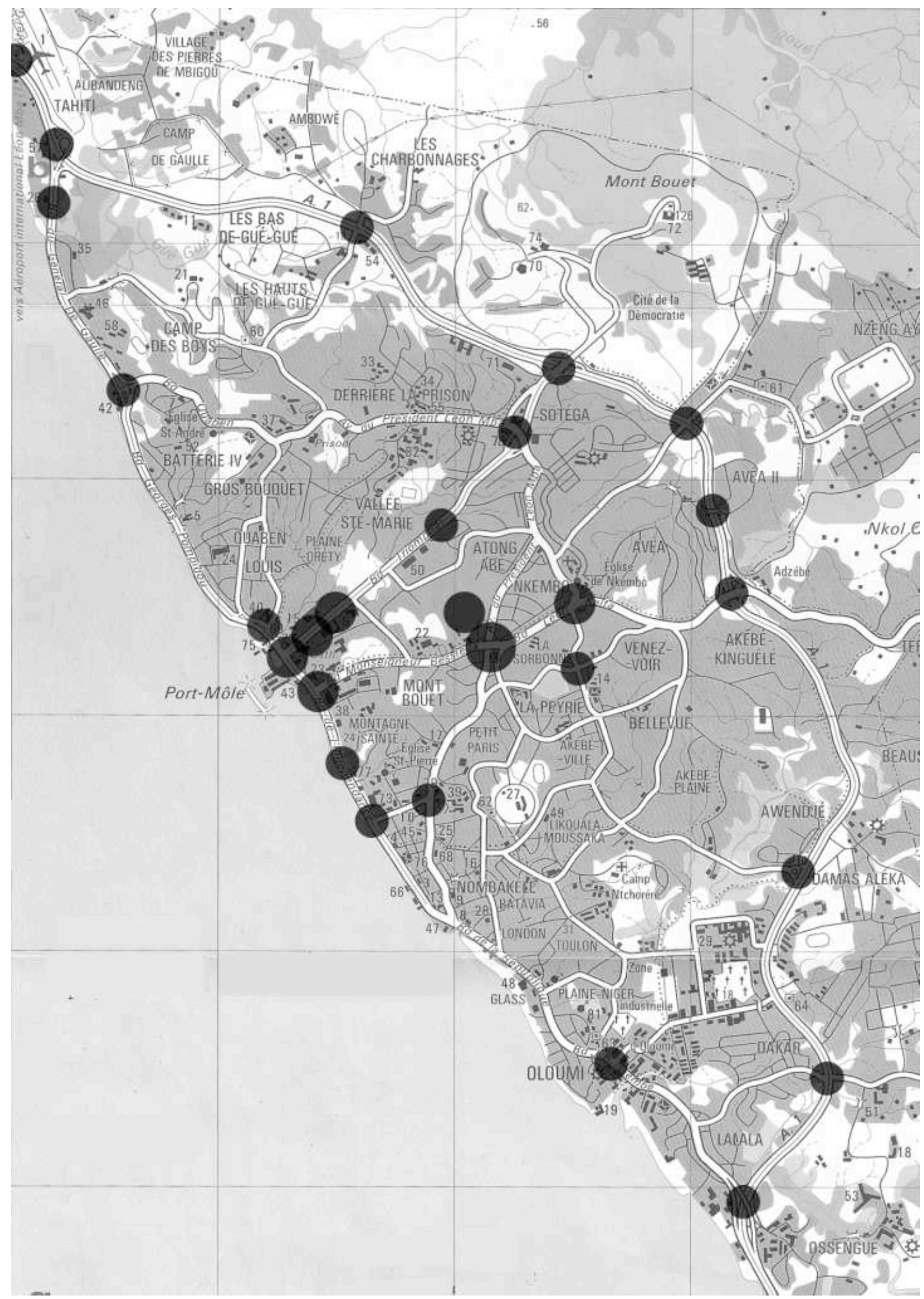

